\title{
1 Pandemic Vibrio cholerae Acquired Competitive Traits from an Environmental Vibrio \\ 2 Species
}

4 Francis J. Santoriello ${ }^{1,4}$, Paul C. Kirchberger ${ }^{2}$, Yann Boucher $^{3}$, and Stefan Pukatzki ${ }^{*}$

$6 \quad{ }^{1}$ Department of Immunology and Microbiology, University of Colorado Anschutz Medical

7 Campus, 13001 East 17th Place, Aurora, CO 80045

$8 \quad{ }^{2}$ Department of Integrative Biology, The University of Texas at Austin, 2506 Speedway, Austin,

$9 \quad \mathrm{TX} 78712$

$10{ }^{3}$ Saw Swee Hock School of Public Health, National University of Singapore, 21 Lower Kent

11 Ridge Rd, Singapore 119077

$12{ }^{4}$ Department of Biology, The City College of New York, 160 Convent Ave, New York, NY,

13 10031, USA

$16{ }^{*}$ Corresponding author

18 Author email addresses:

19 francis.santoriello@cuanschutz.edu

20 pkirchberger@utexas.edu

21 ephyb@nus.edu.sg

22 spukatzki@ccny.cuny.edu 


\section{ABSTRACT}

\section{Background}

28 Vibrio cholerae, the causative agent of cholera, is a human pathogen that thrives in estuarine

29 environments. V. cholerae competes with neighboring microbes by the contact-dependent

30 translocation of toxic effectors with the type VI secretion system (T6SS). Effector types are highly

31 variable across $V$. cholerae strains, but all pandemic isolates encode the same set of distinct

32 effectors. It is possible that acquisition of these effectors via horizontal gene transfer played a role

33 in the development of pandemic V. cholerae.

\section{Results}

35 We assessed the distribution of V. cholerae T6SS loci across multiple Vibrio species. We showed

36 that the fish-pathogen $V$. anguillarum encodes all three $V$. cholerae core loci as well as two of the

37 four additional auxiliary clusters. We further demonstrated that $V$. anguillarum shares T6SS

38 effectors with $V$. cholerae, including every pandemic-associated $V$. cholerae effector. We

39 identified a novel T6SS cluster (Accessory Aux1) that is widespread in V. anguillarum and

40 encodes the pandemic $V$. cholerae effector TseL. We highlighted potential gene transfer events

41 of Accessory Aux1 from $V$. anguillarum to $V$. cholerae. Finally, we showed that TseL from $V$.

42 cholerae can be neutralized by the $V$. anguillarum Accessory Aux1 immunity protein and vice

43 versa, indicating $V$. anguillarum as the donor of tse $L$ to the $V$. cholerae species.

\section{Conclusions}

45 V. anguillarum constitutes an environmental reservoir of pandemic-associated $V$. cholerae T6SS

46 effectors. V. anguillarum and V. cholerae likely share an environmental niche, compete, and

47 exchange T6SS effectors. Further, our findings highlight the fish as a potential reservoir of 48 pandemic $V$. cholerae.

50 KEYWORDS: Vibrio cholerae, Vibrio anguillarum, Type VI Secretion System, mobile DNA, gene 51 transfer 


\section{BACKGROUND}

53 Bacteria live in constant contact with shifting populations of bacterial competitors and predatory

54 eukaryotic cells. Thus, effective niche colonization and survival is often dependent upon the 55 dynamic acquisition of defense mechanisms. One such defense system is the Type VI Secretion 56 System (T6SS), a harpoon-like nanomachine encoded by approximately $25 \%$ of all gram-negative 57 bacteria [1-3]. The T6SS is evolutionarily related to the contractile tail of a T4 bacteriophage [4-

58 7] and is used for the contact-dependent translocation of proteinaceous effectors into neighboring 59 competitor cells (Fig. 1a). Effector proteins can be toxic to non-kin bacteria and eukaryotes [1,8,9].

60 In the case of bactericidal effectors, the effector-secreting cell also encodes a cognate immunity 61 protein to neutralize the effectors killing capacity and protect against attacks from sister cells [10-

62 15]. While this secretion system is functionally conserved across gram-negative species, the core 63 components of the T6SS vary. T6SSs can be phylogenetically classified into four types (T6SS

64 T6SS ${ }^{\mathrm{ii}}, \mathrm{T}_{6 S S^{\mathrm{iii}}}$, and T6SS ${ }^{\mathrm{iv}}$ ) based on multiple independent acquisition events from phages, with

65 T6SS' further divided into six subtypes (i1, i2, i3, i4a, i4b, and i5) [3,16-18].

67 The T6SS was first identified in the main pathogenic lineage of the Vibrio cholerae species [1], 68 and has since been shown to be highly conserved in the Vibrio genus [19-24]. All V. cholerae 69 strains minimally encode their T6SS ${ }^{i 1}$ in three genetic loci $[25,26]$ (Fig. 1b). The Large cluster 70 encodes the majority of the structural components of the system, including the membrane

71 complex that anchors the system to the bacterial inner membrane (vasDFK/tssJLM), the

72 baseplate complex from which the sheath is extended (hsiF/tssE, vasABE/tssFGK), the

73 contractile sheath components $(\operatorname{vip} A B / t s s B C)$, and two tssA-type proteins involved in the

74 regulation of sheath extension and firing (vasJL) [27-29] (Fig. 1a,b). The Large cluster also

75 encodes a VgrG spike (vgrG-3) with a specialized bactericidal C-terminus and its cognate 76 immunity factor (tsiV3) [12]. Two auxiliary T6SS clusters (Aux1 and Aux2) each encode an Hcp 77 protein $(h c p-1,-2)$ that completes the system by forming the central tube upon which the spike is 
78 fired out of the cell, an alternate VgrG spike (vgrG-1, vgrG-2), a chaperone protein for effector

79 loading (tap-1, vasW) [30], and a distinct effector-immunity pair. The VgrG-1 protein encoded by

80 Aux1 is sometimes fused to a specialized C-terminal actin-crosslinking domain (ACD) with anti-

81 eukaryotic properties [4]. Some V. cholerae strains carry additional T6SS loci, of which four have

82 been identified [31-34]. Aux3 minimally encodes an effector-immunity pair (tseH-tsiH) and a

83 PAAR adaptor necessary for its loading onto the T6SS [35], while Aux4, Aux5, and Aux6 encode

84 their own Hcp, VgrG, and chaperone along with effector-immunity pairs. Aux3 and Aux4 are both

85 carried on mobile genetic elements and are transferred between strains [32,36].

87 The $V$. cholerae species is a diverse collection of strains that display varying degrees of virulence

88 to humans, including harmless strains, opportunistic pathogens, and pathogenic strains that have

89 evolved to infect the human gastrointestinal tract. Once ingested into the host gut, some

90 pathogenic $V$. cholerae strains cause the deadly secretory diarrhea known as cholera. These

91 toxigenic strains are primarily defined by the presence of the virulence factors cholera toxin and

92 toxin co-regulated pilus [37-40] and have variable serotypes. Most of these strains belong to the

93 pandemic generating lineage, a monophyletic group of strains descended from an ancestor with

94 the 01 serotype [41]. The first six cholera pandemics were caused by 01 strains of the Classical

95 biotype, and the current $7^{\text {th }}$ pandemic is caused by 01 strains of the El Tor biotype [42-45]. T6SS

96 regulation and composition vary between $\mathrm{O} 1 \mathrm{Classical,} \mathrm{O} 1 \mathrm{EI}$ Tor, and environmental strains of

97 V. cholerae. Most environmental V. cholerae strains constitutively express their T6SS $[46,47]$. For

98 pandemic strains, the $\mathrm{O} 1 \mathrm{El}$ Tor T6SS is tightly regulated by host signals [48], while 01 Classical

99 strains lack a functional T6SS due to mutations in vipA/tssB, hsiF/tssE, vasE/tssK, and vasK/tssM

$100 \quad[9,49]$.

101

102 While the T6SS on a whole is conserved across $V$. cholerae, different strains encode different 103 effectors [24,26]. Environmental V. cholerae strains encode a wide variety of effector types, while 
104 pandemic $V$. cholerae strains all encode an identical set of distinct effectors referred to as A-type

105 (tseL, vasX, vgrG-3) as well as the vgrG-1 ACD (Fig. 1c). It is important to note that different

106 effector genes at the Aux1 (A or C type) and Aux2 (A-E type) locus encode distinct proteins, while

107 variable types at the Large cluster (A-L) are different C-terminal extensions on a conserved VgrG

108 spike [26]. There is no cross protection between types, and any disagreement in effector set

109 composition can lead to intraspecies competition [26] (Fig. 1a,d). Not only are environmental V.

110 cholerae T6SS effector sets highly variable, but T6SS effectors are horizontally transferred

$111[24,50]$. One indication of effector transfer between strains is the maintenance of past immunity

112 genes without their cognate effectors (orphan immunity genes) at the V. cholerae T6SS loci [24].

113 The exact mechanism of recombination that leads to orphan retention is unknown, but we

114 hypothesize that orphan retention is an advantageous evolutionary mechanism that allows a

115 constitutive T6SS-producing strain to exchange an effector-immunity type without becoming

116 vulnerable to surrounding ex-kin cells. The Aux1 locus of pandemic V. cholerae encodes a single

117 orphan C-type immunity gene, leading us to hypothesize that the Aux1 A-type effector/immunity

118 pair (tseL/tsiV1) was acquired by an ancestor of the $V$. cholerae pandemic clade that encoded a

119 C-type effector/immunity pair at Aux1. Acquisition of A-type effectors may have been

120 advantageous for pandemic spread of the clade.

122 In this study, we aimed to identify the evolutionary history of pandemic $V$. cholerae-associated A-

123 type T6SS effectors. We showed that $V$. anguillarum and closely related fish-colonizing Vibrio

124 species ubiquitously encode the Aux1 A-type effector tseL. Further, many V. anguillarum strains

125 also encode the vgrG-1 ACD, vas $X$, and vgrG-3. We indicated likely interaction between $V$.

126 cholerae and $V$. anguillarum in the environment and highlighted potential transfer events of T6SS

127 effectors between the two species. Finally, we showed that the tseL-tsiV1 effector immunity pair

128 from $V$. cholerae and the Aux1 A-type pair from $V$. anguillarum cross-neutralize each other,

129 indicating an evolutionary relationship between the two clusters. This potential niche sharing and 
130 gene flow between $V$. cholerae and $V$. anguillarum may bolster fish as an important environmental

131 reservoir for $V$. cholerae.

132

133 RESULTS

134 All $V$. cholerae T6SS loci with the exception of Aux5 and Aux6 are conserved in a clade of 135 fish-colonizing Vibrio species.

136 While the T6SS is highly conserved across the Vibrio genus, different species encode different 137 subtypes of the T6SS'. We first aimed to identify Vibrio species that encode the core and auxiliary

138 T6SS loci identified in V. cholerae. We compiled a dataset of 247 genomes from V. cholerae (Vch, $139 \mathrm{n}=61), V$. paracholerae (Vpch, $\mathrm{n}=12), V$. metoecus (Vmet, $\mathrm{n}=26)$, . mimicus $($ Vmim, $\mathrm{n}=22), V$.

140 parilis (Vpar, $\mathrm{n}=1)$, V. furnissii (Vfur, $n=8)$, V. fluvialis (Vflu, $\mathrm{n}=18)$, V. anguillarum (Vang, $\mathrm{n}=63)$,

141 V. ordalii (Vord, $n=2)$, V. vulnificus (Vvul, $n=9)$, V. parahaemolyticus $($ Vphl, $n=5)$, V. scophthalmi

142 (Vsco, n=4), V. kanaloae (Vkan, n=12), and closely related Vibrio species (Vibrio sp., $\mathrm{n}=3$ ) and

143 probed for T6SS clusters. Our search identified a single T6SS ${ }^{\mathrm{i1}}$ cluster in all species but Vul.

144 Strains of Vphl (4/5), V. flu (2/18), Vfur (8/8), Vang (39/63), Vch (1/61), and Vmim (1/22) were

145 also found to encode a $\operatorname{T6SS}^{\mathrm{i5}}$ (Fig. 2a,b, Additional File 3). All analyzed Vvul strains encode a

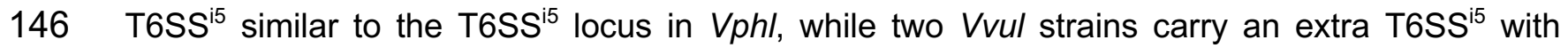
147 homology to the T6SS ${ }^{i 5}$ found in Vflu, Vfur, Vang, Vch, and Vmim (Fig. 2a, Additional File 3). 148 Based on the reported dispersion of the latter T6SS ${ }^{i 5}$ in $V v u l$ [51] and our cross-species analysis, 149 this entire locus is likely horizontally transferred.

151 Next, we identified potential auxiliary clusters (containing some but not all elements of a functional 152 T6SS) in each species. To date, six Auxiliary clusters (Aux1-6) have been identified in Vch (Fig. 153 1b). We performed tblastn searches against our Vibrio genome dataset for vgrG sequences with 154 a $60 \%$ grade cutoff (a weighted score accounting for query coverage, e-value, and pairwise 155 identity) as well as sequences for all known effector types from each Vch T6SS Aux cluster (30\% 
156 grade cutoff for Aux effectors and 60\% grade cutoff for Large effectors) [24,31-34]. The core Vch

157 T6SS loci Aux1 and Aux2 have previously been identified in Vmet, Vmim, Vflu, and Vfur [23,24].

158 Our searches confirmed the Aux1 and Aux2 clusters in these species and identified Aux1 and 159 Aux2 loci in Vang and Vord species (Fig. 2b,c, Additional File 4). In the majority of Vang strains, 160 a region homologous to the Vch Aux1 cluster from vgrG-1 through tsiV-1 is encoded at the end 161 of the Large T6SS ${ }^{i 1}$ cluster (Large/Aux1 Hybrid) while a vgrG-3 homolog is encoded next to the 162 hcp gene usually associated with Aux1 (Aux1/Large Hybrid) (Fig. 2c). These hybrid clusters were 163 likely produced by a recombination event between the conserved 5'-regions of vgrG-1 at the Aux1 164 cluster and vgrG-3 at the Large cluster. To our knowledge, this is the first observation of 165 recombination between regions encoding structural components of two T6SS clusters in a Vibrio 166 genome. The Vang species also encodes Aux3 (2/63) and Aux4 (18/63). Interestingly, Vang and 167 Vord species encode an extra T6SS cluster absent from all other analyzed species. This novel 168 cluster lacks $h c p$ and vgrG genes, encoding only a putative DUF4123-containing T6SS chaperone 169 protein [30], a single effector gene and anywhere from 1 to 7 immunity cassettes (Fig. 2b,c). As 170 this cluster always encodes an Aux1 A-type effector homologous to Vch TseL (Additional File 1: 171 Figure S2), we have named it Accessory Aux1 (Acc Aux1).

173 None of the analyzed Vvul genomes encode T6SS Aux clusters, and only one of the five analyzed $174 V p h l$ genomes (CFSAN018762) encodes an Aux cluster (Aux4). Three of the four available Vsco 175 genomes encode a T6SS, and two of these genomes were found to encode multiple Aux clusters. 176 These two Vsco strains were found to encode an Aux2 (2/2) and an Aux4 (1/2) cluster (Additional 177 File 1: Figure S2). The remaining Aux loci in these strains did not encode effectors with Vch 178 homologs (data not shown) and were thus exclude from downstream analyses. Vsco strain VS17905 was also found to encode Aux4 (Additional File 1: Figure S2), but the Aux4 cluster genes came 180 at the end of an intact T6SS ${ }^{i 1}$ cluster carried on a plasmid (data not shown). Five of the 12 
181 analyzed Vkan genomes encode a T6SS ${ }^{11}$, and each of these strains encodes some combination

182 of Aux1, Aux2, and Aux4 (Additional File 1: Figure S2).

183

184 Cross-species effector gene distribution indicates the presence of pandemic-associated 185 V. cholerae T6SS effectors in the V. anguillarum clade.

186 With our finding that the Vch Aux1, Aux2, and Large loci are core T6SS loci in Vang and Vord, 187 we next aimed to assess the relationship of the VangNord (Vang clade) genomes in our dataset 188 to the selected Vch, Vpch, Vmet, Vpar, and Vmim (Vch clade) genomes. We first constructed a 189 multi-species, core genome phylogeny based on 1,889 core proteins (Fig. 3). The Vang clade 190 branches from a common ancestor that went on to found the Vch and Vflu/Vfur clades, indicating 191 that Vang and Vord are more distant relatives of Vch than are Vflu and Vfur. We next assessed 192 the distribution of known Vch effector types across the selected Vibrio species. We identified Vch 193 effector types in the target genomes by tblastn with known representatives for each effector type 194 using a 30\% grade cutoff for Aux cluster effectors and a 60\% grade cutoff for VgrG effectors. 195 Individual strain effector sets are shown in the expanded phylogenetic trees for each species in 196 the supplement (Additional File 1: Figure S1, S2). The distribution of effector types at the Aux1 197 cluster (Fig. 3) indicates that the C effector-immunity pair is widespread across the Vch, VfluNfur, 198 and Vang clades and was likely encoded by the founding Aux1 cluster in the most recent common 199 ancestor of the three clades. The Aux1 A effector (tse L), however, is irregularly distributed across 200 the three clades, indicating likely horizontal transfer of this effector-immunity module (Fig. 3). 201 Previous work has demonstrated that the Aux1 A effector is highly enriched in the pandemic clade 202 of Vch and is sporadically distributed throughout environmental Vch, Vmet, and Vmim strains [24]. 203 Here we show for the first time that the Aux1 A effector is widespread within the Vang clade. 204 Interestingly, Vang and Vord uniformly encode both and Aux1 A and C effectors. This excess of 205 Aux1 effectors is due to the presence of both a standard Aux1 cluster and the newly identified 206 Acc Aux1 cluster (Fig. 2b,c). 
208 A similar distribution is seen for other pandemic-associated Vch effectors. The vgrG-1 ACD is 209 only observed in Vch, Vpar, and the Vang clade: 25 of 61 Vch strains, 1 of 1 Vpar strains (V. 210 parilis RC586) [52], and 23 of 63 Vang strains (Fig. 3, Additional File 1: Figure S1, S2). At the

211 Aux2 cluster, D- and E-type effectors are present in all three clades, but A- (vasX), B-, and C-type

212 effectors are present only in the Vch and Vang clades (Fig. 3). The A-type effector encoded at 213 the large cluster (vgrG-3), however, is found in the VfluNfur clade as well as the Vch and Vang 214 clades (Fig. 3). Finally, the Aux3 effector/immunity pair (tseH/tsiH), which is enriched in pandemic $215 V$. cholerae strains [36], is restricted to Vch, Vmim, and the Vang clade. It is important to note, 216 that our analysis is restricted by the small number of available Vflu and Vfur genomes. It is 217 possible that the observed lack of pandemic-associated Vch effectors in the VfluNfur clade is due 218 to sampling bias. Due to the presence of the Aux1 A effector (tseL) at a novel T6SS gene cluster 219 that is widespread in the Vang clade, we focused on the evolutionary history of this effector gene 220 for the remainder of the study.

Aux1 A-type T6SS effectors are horizontally transferred between $V$. anguillarum and $V$.

223 cholerae.

224 We next sought to identify horizontal gene transfer events between Vch and the Vang clade. 225 Every identified A-type Aux1 cluster in Vch, Vpch, Vmet, and Vmim also carries at least one C226 type orphan immunity gene, indicating that these strains are derived from one or more Aux1 C 227 ancestors and that the Aux1 A effector was acquired from elsewhere. The Vang group is of 228 particular interest as an Aux1 A donor due to the presence of the Acc Aux1 cluster, which does 229 not encode any C-type orphans and was therefore likely founded with an A-type effector.

231 To identify potential donor Acc Aux1 clusters, we extracted all identified A-type effector and 232 immunity genes from each species and clustered the corresponding amino acid sequences with 
233 a cut-off of $80 \%$. This analysis indicated that the Aux 1 A effector can be grouped into five subtypes

234 (A1-A5) (Additional File 1: Figure S3). Aux1 A2 and A3 are unique to the Vang clade, while A1

235 and A4 are shared between Vch and the Vang clade. The Aux1 A immunity genes are more

236 diverse than their cognate effectors (Additional File 1: Figure S4). To account for this increased

237 amino acid diversity, we lowered our clustering cut-off to $70 \%$. The resulting clusters lead us to

238 further subdivide Aux1 A1 and A2 subtypes into A1a/b and A2a/b, respectively. We next built a

239 single protein phylogeny of the Aux1 A effectors and overlaid the corresponding Aux1 and Acc

240 Aux1 clusters for each strain or group of strains along with their new effector-immunity subtypes

241 (Fig. 4a). From this tree, it is evident that the Aux1 A1a effector-immunity pair found in the Vch

242 and Vpch strains most likely originated from a group of Vang strains.

244 Alternatively, it is possible that Vpch acquired the A1a effector-immunity pair from Vang and

245 subsequently transferred the genes to Vch. A group of Vang and Vord strains (Vang 246 V09_P4A23P171, Vang V04_P4A5T148, Vang V14_P6S14T42, and Vord Q67) encode A1a

247 immunity genes ranging from 89.8 to $91.1 \%$ nucleotide identity and 91.7 to $92.5 \%$ amino acid

248 identity to the pandemic Vch A1a immunity gene (Additional File 1: Figure S4). All of the Vpch

249 strains encoding an Aux1 A1a effector-immunity pair, except Vpch 877-163, encode an A1a

250 immunity gene with $91.7 \%$ nucleotide identity and $89.5 \%$ amino acid identity to the pandemic Vch

251 A1a immunity gene (Additional File 1: Figure S4). Vpch 877-163 encodes an Aux1 A1a effector-

252 immunity pair nearly identical to the pandemic $V$. cholerae Aux1 locus, but we cannot determine

253 the directionality of transfer (Additional File 1: Figure S4). Based on A1a immunity gene homology,

254 we are unable to decern which species was the terminal donor of the Aux1 A1 effector to Vch,

255 but our data support Vang as the initial donor to the Vch clade.

257 All Vmet strains with an Aux1 A effector as well as Vch EM-1676A encode an A4 effector-immunity 258 pair, which most likely originated from a different sub-clade of Vang strains including Vang S2 
2/9, Vang NCTC12159, and Vang FS-238 (Fig. 4a). The Aux1 A1b effector found in the Vchrelated Vibrio sp. 2016V-1062 and Vmim VM223 may have originated from a group of several 261 A1b-encoding Vang strains, including Vang V12_P9A6T4 (Fig. 4a).

263 Recombination between Aux1 and Accessory Aux1 to generate orphan immunity arrays 264 occurred on at least two separate instances.

265 We aimed to use the Aux1 C-type orphan immunity genes present in the Vch clade to identify 266 groups of strains that constitute likely recipients of the Aux1 A effector-immunity module from the 267 Vang clade. To identify potential Aux1 recipient clusters, we extracted all identified C-type effector 268 and immunity genes, including both bona fide (encoded next to their cognate effector) and orphan 269 immunity genes, from each species and clustered the corresponding amino acid sequences with 270 a cut-off of $80 \%$. Like the A-type effectors, we identified five C effector subtypes (C1-C5)

271 (Additional File 1: Figure S5). Again, the cognate immunity genes for two $\mathrm{C}$ subtypes were more 272 variable than their effectors, leading to further subdivisions $(\mathrm{C} 1 \mathrm{a} / \mathrm{b}$ and $\mathrm{C} 2 \mathrm{a} / \mathrm{b})$ (Additional File 1:

273 Figure S6). Unlike the Aux1 A effectors, C effector subtypes are clade specific, with C1 restricted 274 to the Vch clade, C2 restricted to the Vang clade, and C3-5 restricted to the Vflu/Vfur clade.

276 We next overlaid our constructed Aux1 A effector phylogeny with our newly established Aux1 C 277 effector-immunity sub-types (Fig. 4a). Each orphan-encoding Aux1 A cluster in Vch, Vpch, Vmet, 278 or Vmim carries a C1 type orphan. Based on this result, we concluded that on one or more 279 occasions the Acc Aux1 cluster from a Vang strain was transferred to Vch and closely related 280 Vibrio species where it recombined with an Aux1 C1 cluster (Fig. 4b,c, Additional File 1: Figure 281 S7). An alternate scenario in which Acc Aux1 recombined with the Aux1 C2 cluster in the Vang 282 clade to create the orphan-encoding locus we see in Vch is unlikely based on the orphan C 283 immunity subtypes. 
285 The Vch 2012Env-9 Aux1 cluster is a potential predecessor of the pandemic Aux1 locus, as it 286 encodes a bona fide C immunity protein that is $>95 \%$ identical to the orphan $\mathrm{C}$ immunity protein 287 encoded by pandemic strains (Fig. 4b). Vch 2012Env-9 is also a member of a sister clade to the $288 V$ Vh pandemic clade (Fig. 1c) and is thus a good extant relative of the pre-pandemic ancestor that 289 received the Aux1 A effector [24]. Recombination between Acc Aux1 and Aux1 likely occurred by 290 homology-facilitated illegitimate recombination, a known mechanism for interspecies gene 291 transfer [52-55]. An initial crossover occurred in a region of strong homology between the Acc 292 Aux1 DUF4123-containing chaperone and the Aux1 tap-1 chaperone. The second crossover 293 event likely occurred between the 3' end of the donor A1 immunity gene and the 3' end of the C1 294 effector, despite no obvious homology between the two genes, leading to displacement of the C1 295 effector and maintenance of the C1 orphan (Fig. 4b).

297 All Aux1 A4-encoding Vmet strains and Vch EM-1676A encode not only a C1 orphan, but also an

298 A1 type orphan (Fig. 4a) [24]. This array of orphan immunity genes indicates that transfer of the 299 Acc Aux1 A4 effector/immunity pair into Vmet/Vch was likely a second independent transfer event

300 in which the incoming Acc Aux1 A4 cluster recombined into an Aux1 cluster that had already 301 acquired the Aux1 A1 effector/immunity pair (Fig. 4c). There is no obvious recipient Aux1 cluster 302 into which Acc Aux1 A4 recombined to form the Vch EM-1676A Aux1. The Vch EM-1676A Aux1 303 cluster encodes type C1a and A1a orphan immunity genes (Fig. 4a,c) but no vgrG-1 ACD. Based 304 on this, we hypothesize that Acc Aux1 A4 either recombined into an unidentified Aux1 cluster with 305 a matching immunity gene array but no ACD or a pandemic-like Aux1 that later lost its ACD. The 306 necessary gene clusters to back either hypothesis, while likely present in the $V$. cholerae species, 307 are yet to be discovered.

308

309 V. cholerae kills $V$. anguillarum in a T6SS-dependent manner. 
310 We hypothesize that Vch and Vang co-occupy a niche in the aquatic reservoir and compete in a

311 T6SS-dependent manner, leading to the subsequent exchange of genetic material. To test this

312 hypothesis, we assessed whether Vch can kill Vang via its T6SS by competing two strains of Vch

313 with constitutively active T6SSs, the 037 pathogenic strain V52 and the environmental strain

314 DL4211, against a Vang isolate (VIB43). The T6SS effector set of Vang VIB43 (Aux1 C2, Acc

315 Aux1 A1, Aux2 C, Large G) is incompatible with both V52 (Aux1 A1, Aux2 A, Large A) and DL4211

316 (Aux1 C1, Aux2 E, Large E). After 4 hours of co-incubation at $28^{\circ} \mathrm{C}$ on agar at a $1: 1$ ratio, both

317 strains of Vch outcompeted Vang VIB43 (Fig. 5a-d). Importantly, co-incubation of Vang VIB43

318 with Vch carrying an in-frame deletion in the T6SS membrane complex component vasK (V52

$319 \Delta$ vasK and DL4211 $\Delta$ vasK) shows no competitive advantage for either strain (Fig. 5a-d), indicating

320 that this fitness advantage for Vch is T6SS-dependent. In theory, Vang VIB43 should also kill both

321 Vch strains, but our understanding of the T6SS induction conditions for Vang is limited. It is

322 possible that the Vang VIB43 T6SS was not induced under our experimental conditions.

323

324 V. cholerae Aux1 A1a and V. anguillarum Acc Aux1 A1a effector/immunity pairs cross-

325 neutralize.

326 If the Vch Aux1 A1a effector-immunity pair originated from the Vang Acc Aux1 gene cluster, then

327 each immunity gene should be cross-protective against the effector of the opposing cluster. To

328 test this hypothesis, we co-expressed either the A-type effector from Vch N16961 (TseL) or Vang

329 V09_P4A23P171 (Aeff ${ }^{\mathrm{V} 09}$ ), each fused to an N-terminal periplasmic secretion signal, with both

330 their cognate immunity gene and the opposing immunity gene (TsiV1 or Aimm ${ }^{\mathrm{V} 09}$ respectively) in

331 E. coli BL21(DE3) (Fig. 5e). Expressing either TseL or Aeff ${ }^{\mathrm{V} 09}$ alone leads to an approximately

332 10-fold reduction in the number of recovered, viable E. coli cells, while expression of either TsiV1

333 or Aimm ${ }^{\mathrm{V} 09}$ alone had no effect on cell viability (Fig. 5f). Cells co-expressing TseL or Aeff ${ }^{\mathrm{V} 09}$ with

334 their respective cognate immunity genes had no significant reduction in viability. Neither was a 
335 reduction in viability observed when each effector was co-expressed with the opposing immunity

336 gene. These results support our hypothesis that TseL and Aeff ${ }^{\mathrm{V} 09}$ are related effector-immumity

337 clusters that can cross-neutralize each other.

338

339 DISCUSSION

340 Inferring the interaction of $\boldsymbol{V}$. cholerae and $\boldsymbol{V}$. anguillarum in nature

$341 V$. cholerae and $V$. anguillarum are both aquatic organisms that undergo a pathogenic cycle, and

342 it is possible that these two species share common niches. Previous studies have demonstrated,

343 both in-situ and in-silico, that $V$. anguillarum and $V$. cholerae come into contact in the environment.

344 It has been shown experimentally that $V$. cholerae can maintain R-plasmids received via

345 conjugative transfer from $V$. anguillarum [56], indicating that $V$. cholerae and $V$. anguillarum have

346 the potential to share DNA. While R-plasmids have a broad host range, it is interesting to note

347 that $V$. cholerae cannot stably maintain R-plasmids received from Enterobacteraceae [56]. More

348 recently, it was discovered that a genomic island carried in four strains of O1 El Tor V. cholerae

349 is $97 \%$ identical at the nucleotide level to a homolog in V. anguillarum VIB43 [57]. Similar loci

350 have also been identified in multiple strains of non-O1 V. cholerae [58]. Our findings further

351 support the idea that $V$. cholerae interacts with $V$. anguillarum, as we identify specific gene

352 transfer events between the two species. We also show that should $V$. cholerae and $V$.

353 anguillarum come into contact in the environment, V. cholerae can efficiently kill V. anguillarum

354 with its T6SS. The DNA released from lysed $V$. anguillarum cells can then be taken up by $V$.

355 cholerae [59]. This is likely not a one-way interaction, as we show that $V$. anguillarum encodes its

356 own arsenal of T6SS effectors. The $V$. anguillarum T6SS does not appear to be active in strain

357 VIB43 under our experimental conditions, but the $V$. anguillarum T6SSs have been shown to be

358 differentially active under conditions mimicking marine or intra-host conditions and are effective

359 at killing prokaryotic competitors [22,60]. 
361 A niche of interest for the interaction of $V$. cholerae and V. anguillarum is the gut and skin of fish.

362 While $V$. anguillarum is primarily known as a fish pathogen, the incidence of $V$. cholerae colonizing

363 fish in the wild is less understood. It has been shown, however, that both $V$. anguillarum and $V$.

364 cholerae are highly chemotactic towards rainbow trout intestinal mucus [61]. It is possible that the

365 fish gut provides a stable niche for interaction, interspecies killing and gene transfer. Of the 23

366 known $V$. anguillarum serotypes, only $\mathrm{O} 1, \mathrm{O} 2$, and $\mathrm{O} 3$ cause vibriosis in fish [62]. The remaining

367 serotypes are comprised of mostly non-pathogenic environmental isolates from sediment,

368 phytoplankton, and zooplankton. The chitinous exoskeleton of zooplankton is also a niche of

369 interest for the interaction of $V$. cholerae and V. anguillarum, as chitin metabolism by $V$. cholerae

370 induces both the T6SS and the natural competence machinery $[59,63]$. Less is known about

371 natural competence in $V$. anguillarum, but isolates of this species have been shown to encode

372 chitinase and can grow with chitin and $\mathrm{N}$-acetylglucosamine as their sole carbon source [64]. Co-

373 colonization of zooplankton by $V$. cholerae and $V$. anguillarum could result in bi-directional killing

374 and genetic exchange. Our results do not favor either niche as the site of interaction and gene

375 transfer between these two species. We have shown here, however, that a pre-pandemic $V$.

376 cholerae strain carrying an Aux1 C1 effector potentially acquired the Aux1 A1 effector from V.

377 anguillarum, likely as a result of physical contact in the past.

379 Our results as well as previous data from Kirchberger et al., 2017 [24] show that tse L is found in

380 a small number of environmental $V$. cholerae strains and several $V$. paracholerae strains.

381 Approximately half of the analyzed $V$. paracholerae strains encode an A1 effector protein that is

382 closely related to the A1 effector found in pandemic V. cholerae (Fig. 4a, Additional File 1: Figure

383 S1). As V. cholerae and V. paracholerae strains have been isolated from common water sources,

384 an alternate hypothesis to direct interaction between pre-pandemic $V$. cholerae and $V$.

385 anguillarum is gene transfer between $V$. anguillarum and $V$. paracholerae and subsequent

386 transfer to the $V$. cholerae pre-pandemic ancestor. Regardless of which species constitutes the 
387 terminal tse L donor, our orphan $\mathrm{C}$ immunity data supports $\mathrm{V}$. anguillarum as the origin of the A-

388 type effector-immunity pair in Vch and Vpch.

390 Potential of the fish as a niche for pandemic $V$. cholerae

391 V. cholerae, including some toxigenic 01 strains, has been isolated from 30 different species of

392 fish [65]. Fish colonization is likely to provide $V$. cholerae with benefits, such as protection from

393 the stresses of the aquatic environment and dissemination across long distances both by the fish

394 harboring the bacteria and the birds that eat the fish $[66,67]$. In the laboratory, the zebrafish gut

395 is a hospitable environment for $V$. cholerae, as the bacterium readily colonizes the intestine,

396 attaches to the epithelium, and forms microcolonies [68,69]. O1 El Tor infection of the zebrafish

397 leads to a sustained colonization, while infection with 01 Classical strains or non-O1/non-O139

398 environmental strains leads to transient colonization $[68,69]$. Further, expression of major human

399 virulence factors CT and TCP appear to be dispensable for zebrafish gut colonization [68]. The

400 T6SS could play a role in colonization, as 01 Classical strains of $V$. cholerae carry an inactive

401 T6SS $[9,49]$ and O1 El Tor strains should express their pathoadaptive T6SS in the fish gut.

402 Interestingly, infection with V. cholerae strain AM-19226 (non-O1/non-O139), which constitutively

403 expresses its T6SS, shows a similar transient colonization pattern to 01 classical strains [69],

404 indicating that there is more to fish colonization than simply having an active T6SS. A single study

405 has directly investigated the $V$. cholerae T6SS in the fish gut. In this work, an O1 El Tor strain that

406 was modified to constitutively express the T6SS was shown to displace the resident fish gut

407 microbes in a vgrG-1 ACD dependent manner [70]. This is not sufficient to explain colonization of

408 the fish gut by $\mathrm{O} 1 \mathrm{El}$ Tor strains, however, because environmental strain AM-19226 also

409 constitutively expresses its T6SS and encodes the vgrG-1 ACD [24]. We believe that our results

410 warrant investigation of the role of different T6SS effector types, specifically pandemic-associated

411 T6SS effectors like TseL, in fish colonization by $V$. cholerae. Improved colonization of the fish gut

412 could lead to increased dissemination and potentially adaptation to a host intestinal tract. 


\section{T6SS effector gene evolution by interspecies transfer and its role in pandemicity}

415 We show that $V$. cholerae is sharing genetic material, specifically pandemic-associated T6SS

416 effectors with $V$. anguillarum and V. ordalii. Common T6SS effectors is likely an indication of a

417 shared niche because acquiring effector/immunity sets similar to neighboring cells is a protective

418 strategy [50]. Here we show that, within our dataset, the V. cholerae T6SS Aux1 A-type effector

$419 t$ tse $L$ is uniquely shared between the $V$. cholerae and $V$. anguillarum clades. It is important to note

420 that $V$. anguillarum is more extensively sampled than $V$. fluvialis/ $V$. furnissii, so we cannot rule

421 out sampling bias in the observed distribution of TseL. Our data show that, on at least two

422 occasions, A-type effectors originating in the V. anguillarum Acc Aux1 cluster were transferred to

423 the V. cholerae clade (Fig. 6). In the first transfer event, an A1a effector-immunity module was

424 likely acquired by the $V$. cholerae pre-pandemic ancestor, displacing its $\mathrm{C} 1$ effector gene (Fig.

425 6a). In the second transfer event, an A4 effector-immunity module displaced the A1 effector (Fig.

426 6a). Based on the diversity of orphan $\mathrm{C}$ immunity arrays observed at Aux1 in V. cholerae, V.

427 paracholerae, V. metoecus, and V. mimicus, it is likely that more than two transfers of the Aux 1

428 A effector occurred between the two clades (Fig. 6b).

430 If $V$. cholerae shares niches and DNA with both $V$. fluvialis/V. furnissii and $V$. anguillarum but only

431 exchanges the Aux1 A T6SS effector with $V$. anguillarum, then it is possible that this effector is

432 adaptive to the shared niche of $V$. cholerae and $V$. anguillarum. This raises the question of

433 whether TseL plays an environmental role, as pandemic-associated $V$. cholerae genomic islands

434 have been shown to have environmental significance [71]. One potential environmental role of

435 Tsel could be fish gut colonization, which in turn could favor pandemicity in some way. For

436 instance, the VgrG-1 ACD has been shown to induce intestinal inflammation in the mouse model

437 of infection $[8,72]$ and expedite niche clearance in the fish gut through the induction of peristalsis

438 [70]. This is a clear example of a T6SS effector that is beneficial for both fish gut colonization and 
mammalian disease. Based on our in-silico results showing that TseL is widespread in the $V$. anguillarum clade, we hypothesize that a parallel scenario may exist for the Aux1 A effector. We

441 cannot, however, restrict the potential benefits of TseL to fish gut colonization, as TseL is present

442 in both vibriosis-causing and non-pathogenic, environmental $V$. anguillarum isolates.

\section{CONCLUSION}

445 In this study, we assessed the distribution of T6SS loci and effector types across 13 Vibrio species

446 to reconstruct the evolutionary history of the pandemic $V$. cholerae T6SS and to indicate potential

447 interspecies interactions. Our genomic results indicated sharing of T6SS effector/immunity

448 modules between $V$. anguillarum and $V$. cholerae and lead us to conclude that these two species

449 may share one or more aquatic niches and regularly compete. We propose that $V$. anguillarum

450 constitutes an environmental reservoir of pandemic-associated $V$. cholerae T6SS effectors,

451 indicating that the pandemic A-type effectors may play important roles outside of human

452 pathogenesis. Our findings highlight fish colonization as a potential stage during the evolution of

453 pandemic $V$. cholerae and indicate that competitive fitness in the fish gut may be important for

454 pandemic $V$. cholerae strains in the environmental reservoir.

\section{METHODS}

457 Acquisition and annotation of publicly available genome sequences

458 All genome sequences used in this study are publicly available and were downloaded from the 459 NCBI GenBank database. GenBank Accession numbers for all strains are listed in Additional File 460 2: Table S1. A single fasta file was generated for each genome and all files were re-annotated 461 with Prokka (v1.12) [73] for uniformity across genomes. 
464 Complete T6SS clusters were identified using HMmer Based UndeRstandinG of gene clustERs

465 (hamburger)(https://github.com/djw533/hamburger). GFF files generated with Prokka were

466 analyzed with hamburger using the -t option for an automatic T6SS search based on 13 highly

467 conserved structural and regulatory genes. Hamburger then aligned the identified T6SS clusters

468 with an internal reference set of identified T6SS cluster types $\left(\right.$ T6SS $^{i 1}-\mathrm{T} \mathrm{SS} \mathrm{S}^{\mathrm{i}}$ ) to assign types

469 to the extracted loci. This alignment was then used by hamburger to generate a phylogenetic tree

470 of T6SS loci with corresponding gene diagrams aligned around the vipA/vipB (tssB/tssC)

471 cassettes.

\section{Identification of Auxiliary T6SS clusters}

474 Auxiliary T6SS clusters were not identified by hamburger as they do not encode a sufficient

475 number of T6SS genes to be considered a contiguous T6SS cluster and subsequently extracted

476 by the program using the -t option. Hamburger does not identify effector and immunity gene

477 cassettes as these are extremely variable both between and within species. For these reasons,

478 auxiliary clusters were identified manually from our custom database of 247 genomes. Further,

479 several genomes in this study have only been assembled into contigs, occasionally resulting in

480 fragmented T6SS loci. As there is no one method that will capture every loci under these

481 conditions, we use the following three methods. Vibrio auxiliary clusters commonly encode vgrG

482 genes, and so auxiliary clusters were identified in an unbiased manner by performing a tblastn

483 search for vgrG-2 (VCA0018) from V. cholerae N16961 with a Geneious grade (a weighted metric

484 combining query coverage (0.50), e-value (0.25), and pairwise identity $(0.25)$ ) cutoff of $60 \%$.

485 Auxiliary clusters were also identified in a biased manner by performing a tblastn search for all

486 known T6SS effector types from V. cholerae with a Geneious grade cutoff of $30 \%$ for effectors

487 associated with Auxilliary clusters and a Geneious grade cutoff of $60 \%$ for the VgrG effectors of

488 the Large cluster [24,31-34]. The N-terminal VgrG portion of all V. cholerae Large cluster effector

489 types is highly conserved, and thus these effector types require a higher grade cutoff to 
490 differentiate. Finally, once auxiliary loci had been identified in each species, flanking, non-T6SS

491 genes were used to find any missing loci in other strains of that species. The identified auxiliary

492 loci were verified against the literature to separate known and novel T6SS loci [23,24]. All

493 identified loci were extracted from their respective genomes for downstream analyses. As this

494 study focuses on $V$. anguillarum, the genomic locus of all identified T6SS auxiliary clusters from

$495 \quad$ V. anguillarum strains are listed in Additional File 4.

496

497 Core genome and single protein phylogeny

498 A species level phylogeny (Fig. 3, Fig. 6a) was constructed as follows: The core genome of 30

499 Vibrio strains (Additional File 2: Table S2) corresponding to 13 described species, including V.

500 paracholerae, an emerging new species/subspecies closely related to $V$. cholerae, was extracted

501 using usearch with an amino acid cutoff of $30 \%$ and then aligned using MUSCLE [74,75], as

502 implemented by the BPGA pipeline [76]. The resulting alignment of 1,889 core proteins,

503 corresponding to 650,474 amino acid positions, was then used to construct a phylogenetic tree

504 using the GAMMA+WAG substitution model in RAxML (v8.0.26) [77]. Branch support was

505 calculated using 100 bootstrap replicates. Phylogenetic tree was visualized from RAxML-

506 generated newick file using TreeGraph 2 (v2.15.0-887 beta) [78]. Branches with bootstrapping

507 support values $<70$ were collapsed.

508

509

510 Core genome based phylogenetic trees for individual clades and species (Additional File 1: Figure

511 S1, S2) were constructed as follows: Annotated GFF3 files generated by Prokka (v1.12) [73] were

512 used for this analysis. Core genes were extracted from the GFF3 files based on their translated

513 amino acid sequence using a cd-hit cutoff for homologous proteins of $95 \%$, and extracted genes

514 were aligned using Roary (v3.11.2) [79]. The core genome alignment was reduced to only loci

515 harboring polymorphisms using SNP-sites (v2.4.1) [80]. A Maximum Likelihood phylogenetic tree 
516 was built using RAxML [77] with the GTR+Gamma model. Statistical branch support was obtained

517 from 100 bootstrap repeats. Phylogenetic trees were visualized from RAxML-generated newick

518 files using TreeGraph 2 (v2.15.0-887 beta) [78]. Branches with bootstrapping support values $<70$

519 were collapsed.

520

521 Single protein phylogenetic trees were constructed as follows: Nucleotide sequences for genes

522 of interest were extracted from annotated genomes and translated in Geneious (v2019.0.4). Only

523 full length sequences were used to generate single protein phylogenetic trees. Any partial or

524 truncated protein sequences were discarded. Protein sequences were aligned using Muscle

525 (v3.8.425) [74]. Pairwise Muscle alignments were used for tree building with RAxML as described

526 above. Phylogenetic trees were visualized with TreeGraph 2 (v2.15.0-887 beta) [78]. Branches

527 with bootstrapping support values $<70$ were collapsed.

528

529

T6SS effector and immunity protein typing and subtyping

530 Effector/immunity protein typing: All sequence manipulations were performed in Geneious

531 (v2019.0.4). All effector and immunity gene sequences were extracted from all identified Aux1

532 and Accessory Aux1 clusters from all analyzed species. All effectors and immunity nucleotide

533 sequences were translated. Blastp was performed against a custom effector or immunity gene

534 database generated by Kirchberger et al., 2017 [24] for each amino acid sequences to identify

535 effector or immunity type (Ex: A, B, C, etc.). For a given sequence, the strongest hit over $30 \%$

536 identity was considered its type [24,26].

537

538 Effector/immunity protein subtyping: All effector or immunity amino acid sequences for a given

539 type were aligned with MUSCLE (v3.8.425) [74] to generate a pairwise amino acid identity matrix.

540 Only full-length sequences were used for this analysis. Clusters were determined by inputting

541 protein fasta files to CD-HIT $[81,82]$ with an $80 \%$ identity clustering cut off for effector proteins 
542 (ex: A1, A2, A3, etc) and a 70\% cutoff for immunity genes (ex: A1a, A1b). An 80\% identity cutoff

543 for effector proteins is based on experimental data showing a loss of neutralization between two

544 effector/immunity pairs of the same type below $80 \%$ identity. Immunity proteins were more

545 variable than effector proteins and thus the identity cutoff was dropped to $70 \%$ to be conservative

546 in our typing scheme. Pairwise matrices were used to generate heatmaps using pheatmap

547 (pheatmap, R package pheatmap v1.0.12).

548

549 Alignment of T6SS clusters

550 Full length T6SS clusters were aligned using the Artemis Comparison Tool (ACT, v18.1.0) [83].

551 Nucleotide sequences were submitted to NCBI blastn with the "align two or more sequences"

552 option. Alignment files were exported from NCBI and input to ACT along with GenBank format

553 files of the aligned regions.

554

\section{Bacterial Strains, Plasmids, and Growth Conditions}

556 V. cholerae strains, V. anguillarum strains, E. coli strains, plasmids, and primers used in this study

557 are listed in Additional File 2: Tables S3 and S4. E. coli strain DH5a $\lambda$ pir was used for cloning. All

558 E. coli strains were routinely cultured at $37^{\circ} \mathrm{C}$ in Lysogeny Broth - Lennox (LB) with shaking at

$559250 \mathrm{rpm}$. Culture on agar plates was done on LB agar at $28^{\circ} \mathrm{C}$ (V. cholerae, V. anguillarum, and

560 E. coli). When required IPTG (for the induction of the $\mathrm{T} 7$ polymerase), $\mathrm{NaCl}$ (for $\mathrm{V}$. anguillarum

561 culture), or antibiotics were added to liquid or agar culture medium at the following concentrations:

$562100 \mu \mathrm{M}$ IPTG, $2 \% \mathrm{w} / \mathrm{v} \mathrm{NaCl}, 100 \mu \mathrm{g} / \mathrm{mL}$ streptomycin, $50 \mu \mathrm{g} / \mathrm{mL}$ rifampicin, $50 \mu \mathrm{g} / \mathrm{mL}$ kanamycin,

$563100 \mu \mathrm{g} / \mathrm{mL}$ ampicillin, and $30 \mu \mathrm{g} / \mathrm{mL}$ chloramphenicol.

565 Bacterial Strain and Plasmid Construction

566 For all pET vectors, vectors were cut with Xhol and BamHI. Effector or immunity genes were 567 amplified without their stop codon and inserted by Gibson cloning. Primers were modified to 
568 ensure that the start codon was in frame with the upstream start codon of the periplasmic

569 localization signal sequence (pelB). For all genes, primers were modified to add a triple glycine

570 linker between the end of the inserted gene and the vector-encoded $6 x-H i s$ tag.

\section{1}

\section{Competitive killing assays}

573 Predator V. cholerae (streptomycin resistant) and prey V. anguillarum (rifampicin resistant) strains

574 were cultured as lawns on lysogeny broth (LB) agar plates with $2 \% \mathrm{w} / \mathrm{v} \mathrm{NaCl}$ and selective

575 antibiotics. Plates were incubated for $18-20 \mathrm{hr}$ at $28^{\circ} \mathrm{C}$. Predator and prey cells were collected

576 from the overnight lawns with a sterile loop and resuspended in LB $+2 \% \mathrm{NaCl}$ to an optical density

577 at $600 \mathrm{~nm}\left(\mathrm{OD}_{600}\right)$ of 1 . Input cell counts were determined by plating 10 -fold serial dilutions of

578 single strain cell suspensions on either LB agar $+2 \% \mathrm{NaCl}+$ rifampicin or LB agar $+2 \% \mathrm{NaCl}+$

579 streptomycin plates. Predator and prey cells were mixed at a 1:1 ratio, and $25 \mu \mathrm{L}$ of each mixture

580 was spotted on LB agar $+2 \% \mathrm{NaCl}$. Competitive mixture spots were incubated for $4 \mathrm{hr}$ at $28^{\circ} \mathrm{C}$.

581 Spots were harvested by excision of the underlying agar and vortexing in $1 \mathrm{~mL} L B+2 \% \mathrm{NaCl}$.

582 For each killing assay, 10-fold serial dilutions were prepared from output cell suspensions, and

583 dilution series were plated on both LB agar $+2 \% \mathrm{NaCl}+$ rifampicin and $\mathrm{LB}$ agar $+2 \% \mathrm{NaCl}+$

584 streptomycin plates. Input and output plates were incubated for $18-20 \mathrm{hr}$ at $28^{\circ} \mathrm{C}$. Input and output

585 CFU counts were determined for predator and prey strains from each killing assay. Competitive

586 indices were determined as follows: C.I. $=\left[\left(\right.\right.$ Output CFU/mL ${ }^{\text {PREDATOR}} /$ Output $\left.\mathrm{CFU} / \mathrm{mL}^{\text {PREY }}\right) /($ Input

$\left.\left.587 \mathrm{CFU} / \mathrm{mL}^{\mathrm{PREDATOR}} / \mathrm{Input} \mathrm{CFU} / \mathrm{mL}^{\mathrm{PREY}}\right)\right]$.

588

589 Effector/Immunity Co-expression Viability Assays

590 E. coli BL21 (DE3) pLysS strains carrying all pairwise combinations of vectors from the following

591 two lists were generated: (1) pET26b, pET26b-tseL, or pET26b-Aeff ${ }^{\mathrm{V} 09}$ and (2) pET22b, pET22b-

592 tsiV1, or pET22b-Aimm ${ }^{\mathrm{V} 9}$. E. coli strains were grown $\left(18-20 \mathrm{hr}, 37^{\circ} \mathrm{C}\right.$, and $250 \mathrm{rpm}$ shaking) in 1 
$593 \mathrm{~mL}$ LB with selective antibiotics for all three vectors: $30 \mu \mathrm{g} / \mathrm{mL}$ chloramphenicol, $50 \mu \mathrm{g} / \mathrm{mL}$

594 kanamycin, and $100 \mu \mathrm{g} / \mathrm{mL}$ ampicillin. $\mathrm{OD}_{600}$ was calculated, and each overnight culture was

595 normalized to an $\mathrm{OD}_{600}$ of 1 in fresh LB. Normalized cell suspensions were spotted $(10 \mu \mathrm{L})$ on LB

596 agar + chloramphenicol + kanamycin + ampicillin both with and without $100 \mu$ M IPTG (inducing

597 and non-inducing, respectively). Spot plates were incubated for $18-20 \mathrm{hr}$ at $28^{\circ} \mathrm{C}$. Spots from

598 inducing and non-inducing plates were collected by scraping and resuspended in $1 \mathrm{~mL}$ LB by

599 vortexing. Output cell counts were determined by plating 10-fold serial dilutions of each cell

600 suspension on LB agar + chloramphenicol + kanamycin + ampicillin. Output dilution plates were

601 incubated for $18-20 \mathrm{hr}$ at $28^{\circ} \mathrm{C}$. Viability indices were calculated as follows: Viability Index = $602\left[\left(\right.\right.$ Output CFU/mL $\left.\mathrm{mL}^{+\mathrm{IPTG}}\right) /\left(\right.$ Output CFU/mL $\left.\left.{ }^{-\mathrm{IPTG}}\right)\right]$.

603

604 DECLARATIONS

605 Ethics approval and consent to participate

606 Not applicable.

607

608 Consent for publication

609 Not applicable.

610

611 Availability of data and materials

612 Accession codes for all publicly available genomes analyzed in this study can be found in

613 Additional file 2: Table S1. All analyses are performed with publicly-available tools referenced in

614 the Methods. Custom protein databases referred to in the text are available upon request.

616 Competing interests

617 The authors declare that they have no competing interests. 


\section{Funding}

620 This study was funded by the National Institutes of Health (R01Al139103).

\section{Authors' contributions}

623 FJS, PCK, YB, SUP designed the study. FJS and PCK performed analyses. FJS wrote the 624 manuscript. All authors read and approved of the final manuscript.

625

\section{Acknowledgements}

627 We thank Hans Rediers and Daniele Provenzano for the strains and reagents necessary for the 628 completion of this study.

629

\section{Additional Files}

631 Additional File 1 (.pdf): Supplementary Figures. Figures S1-S7 supporting the data presented in 632 the main manuscript.

634 Additional File 2 (.pdf): Supplementary Tables. Tables S1-S4 providing information on all genome 635 files, bacterial strains, and primer pairs used to generate the presented data.

637 Additional File 3 (.xls): T6SS Cluster Information. Table describing the type and location of all 638 T6SS large clusters identified from all analyzed genomes.

640 Additional File 4 (.xls): Vang T6SS Auxiliary Clusters. Table describing the location of all Auxiliary

641 T6SS loci identified from all analyzed $V$. anguillarum genomes.

\section{REFERENCES}


644 1. Pukatzki S, Ma AT, Sturtevant D, Krastins B, Sarracino D, Nelson WC, et al. 645 Identification of a conserved bacterial protein secretion system in Vibrio cholerae using 646 the Dictyostelium host model system. Proceedings of the National Academy of 647 Sciences. 2006;103:1528-33.

648 2. Bingle LE, Bailey CM, Pallen MJ. Type VI secretion: a beginner's guide. Curr Opin 649 Microbiol. 2008;11:3-8.

650 3. Boyer F, Fichant G, Berthod J, Vandenbrouck Y, Attree I. Dissecting the bacterial type VI secretion system by a genome wide in silico analysis: what can be learned from

652 available microbial genomic resources? BMC Genomics. 2009;10:104.

653 4. Pukatzki S, Ma AT, Revel AT, Sturtevant D, Mekalanos JJ. Type VI secretion system 654 translocates a phage tail spike-like protein into target cells where it cross-links actin.

655 Proceedings of the National Academy of Sciences. 2007;104:15508-13.

656 5. Leiman PG, Basler M, Ramagopal UA, Bonanno JB, Sauder JM, Pukatzki S, et al. Type VI secretion apparatus and phage tail-associated protein complexes share a common evolutionary origin. Proceedings of the National Academy of Sciences.

659 2009;106:4154-9.

660 6. Pell LG, Kanelis V, Donaldson LW, Lynne Howell P, Davidson AR. The phage major tail protein structure reveals a common evolution for long-tailed phages and the type $\mathrm{VI}$ bacterial secretion system. Proceedings of the National Academy of Sciences.

663 2009;106:4160-5.

7. Basler M, Pilhofer M, Henderson GP, Jensen GJ, Mekalanos JJ. Type VI secretion requires a dynamic contractile phage tail-like structure. Nature. 2012;483:182-6.

666 8. Ma AT, McAuley S, Pukatzki S, Mekalanos JJ. Translocation of a Vibrio cholerae type VI secretion effector requires bacterial endocytosis by host cells. Cell Host Microbe.

668 2009;5:234-43. secretion system displays antimicrobial properties. Proceedings of the National Academy of Sciences. 2010;107:19520-4.

672 10. Hood RD, Singh P, Hsu F, Güvener T, Carl MA, Trinidad RRS, et al. A Type VI 673 Secretion System of Pseudomonas aeruginosa Targets a Toxin to Bacteria. Cell Host \& 674 Microbe. 2010;7:25-37.

675 11. Russell AB, Hood RD, Bui NK, LeRoux M, Vollmer W, Mougous JD. Type VI 676 secretion delivers bacteriolytic effectors to target cells. Nature. 2011;475:343-7.

677 12. Brooks TM, Unterweger D, Bachmann V, Kostiuk B, Pukatzki S. Lytic Activity of the 678 Vibrio cholerae Type VI Secretion Toxin VgrG-3 Is Inhibited by the Antitoxin TsaB. J Biol 679 Chem. 2013;288:7618-25. 
680 13. Dong TG, Ho BT, Yoder-Himes DR, Mekalanos JJ. Identification of T6SSdependent effector and immunity proteins by Tn-seq in Vibrio cholerae. Proceedings of the National Academy of Sciences. 2013;110:2623-8. Identification of Novel Secreted Antibacterial Toxins of the Serratia marcescens Type VI Secretion System. Molecular \& Cellular Proteomics. 2013;12:2735-49.

686 15. Miyata ST, Unterweger D, Rudko SP, Pukatzki S. Dual Expression Profile of Type VI Secretion System Immunity Genes Protects Pandemic Vibrio cholerae. Mougous JD, 688 editor. PLoS Pathog. 2013;9:e1003752.

16. Barret M, Egan F, O'Gara F. Distribution and diversity of bacterial secretion systems across metagenomic datasets: Secretion systems in metagenomes. Environmental Microbiology Reports. 2013;5:117-26.

692 17. Russell AB, Wexler AG, Harding BN, Whitney JC, Bohn AJ, Goo YA, et al. A Type 693 VI Secretion-Related Pathway in Bacteroidetes Mediates Interbacterial Antagonism.

694 Cell Host \& Microbe. 2014;16:227-36. architecture, function, and evolution of a contractile injection system. Science. 2017;357:713-7.

19. Weber B, Hasic M, Chen C, Wai SN, Milton DL. Type VI secretion modulates quorum sensing and stress response in Vibrio anguillarum. Environmental Microbiology. 2009;11:3018-28.

701 20. Yu Y, Yang H, Li J, Zhang P, Wu B, Zhu B, et al. Putative type VI secretion systems of Vibrio parahaemolyticus contribute to adhesion to cultured cell monolayers. Arch

703 Microbiol. 2012;194:827-35.

704 21. Church SR, Lux T, Baker-Austin C, Buddington SP, Michell SLI. Vibrio vulnificus 705 Type 6 Secretion System 1 Contains Anti-Bacterial Properties. Zhou D, editor. PLoS 706 ONE. 2016;11:e0165500.

707 22. Tang L, Yue S, Li G-Y, Li J, Wang X-R, Li S-F, et al. Expression, secretion and bactericidal activity of type VI secretion system in Vibrio anguillarum. Arch Microbiol.

709 2016;198:751-60.

23. Huang Y, Du P, Zhao M, Liu W, Du Y, Diao B, et al. Functional Characterization and Conditional Regulation of the Type VI Secretion System in Vibrio fluvialis. Front Microbiol [Internet]. 2017 [cited 2020 Jul 26];8. Available from: http://journal.frontiersin.org/article/10.3389/fmicb.2017.00528/full

714 24. Kirchberger PC, Unterweger D, Provenzano D, Pukatzki S, Boucher Y. Sequential displacement of Type VI Secretion System effector genes leads to evolution of diverse immunity gene arrays in Vibrio cholerae. Sci Rep. 2017;7:45133. 
25. Pukatzki S, McAuley SB, Miyata ST. The type VI secretion system: translocation of effectors and effector-domains. Current Opinion in Microbiology. 2009;12:11-7.

26. Unterweger D, Miyata ST, Bachmann V, Brooks TM, Mullins T, Kostiuk B, et al. The Vibrio cholerae type $\mathrm{VI}$ secretion system employs diverse effector modules for intraspecific competition. Nat Commun. 2014;5:3549. Architecture and assembly of the Type VI secretion system. Biochim Biophys Acta.

724 2014;1843:1664-73.

725

726

727

728

729

730

731

732

733

734

735

736

737

738

739

740

741

742

743

744

745

746

747

748

749

750

28. Cianfanelli FR, Monlezun L, Coulthurst SJ. Aim, Load, Fire: The Type VI Secretion System, a Bacterial Nanoweapon. Trends in Microbiology. 2016;24:51-62.

29. Schneider JP, Nazarov S, Adaixo R, Liuzzo M, Ringel PD, Stahlberg H, et al. Diverse roles of TssA-like proteins in the assembly of bacterial type VI secretion systems. EMBO J. 2019;38:e100825.

30. Unterweger D, Kostiuk B, Ötjengerdes R, Wilton A, Diaz-Satizabal L, Pukatzki S. Chimeric adaptor proteins translocate diverse type $\mathrm{VI}$ secretion system effectors in Vibrio cholerae. EMBO J. 2015;34:2198-210.

31. Altindis E, Dong T, Catalano C, Mekalanos J. Secretome Analysis of Vibrio cholerae Type VI Secretion System Reveals a New Effector-Immunity Pair. Rappuoli R, editor. mBio. 2015;6:e00075-15.

32. Labbate M, Orata FD, Petty NK, Jayatilleke ND, King WL, Kirchberger PC, et al. A genomic island in Vibrio cholerae with VPI-1 site-specific recombination characteristics contains CRISPR-Cas and type VI secretion modules. Sci Rep. 2016;6:36891.

33. Crisan CV, Chande AT, Williams K, Raghuram V, Rishishwar L, Steinbach G, et al. Analysis of Vibrio cholerae genomes identifies new type VI secretion system gene clusters. Genome Biol. 2019;20:163.

34. Drebes Dörr NC, Blokesch M. Interbacterial competition and anti-predatory behaviour of environmental Vibrio cholerae strains. Environ Microbiol. 2020;22:4485504.

35. Hersch SJ, Watanabe N, Stietz MS, Manera K, Kamal F, Burkinshaw B, et al. Envelope stress responses defend against type six secretion system attacks independently of immunity proteins. Nat Microbiol. 2020;5:706-14.

36. Santoriello FJ, Michel L, Unterweger D, Pukatzki S. Pandemic Vibrio cholerae shuts down site-specific recombination to retain an interbacterial defence mechanism. Nat Commun. 2020;11:6246. 
37. Cassel D, Selinger Z. Mechanism of adenylate cyclase activation by cholera toxin: Inhibition of GTP hydrolysis at the regulatory site. Proceedings of the National Academy of Sciences. 1977;74:3307-11.

38. Gill DM, Meren R. ADP-ribosylation of membrane proteins catalyzed by cholera toxin: basis of the activation of adenylate cyclase. Proceedings of the National Academy of Sciences. 1978;75:3050-4.

757 39. Herrington DA, Hall RH, Losonsky G, Mekalanos JJ, Taylor RK, Levine MM. Toxin, 758 toxin-coregulated pili, and the toxR regulon are essential for Vibrio cholerae

759 pathogenesis in humans. The Journal of Experimental Medicine. 1988;168:1487-92.

760 40. Thelin KH, Taylor RK. Toxin-coregulated pilus, but not mannose-sensitive hemagglutinin, is required for colonization by Vibrio cholerae O1 El Tor biotype and O139 strains. Infection and immunity. 1996;64:2853-6.

763 41. Islam MT, Alam M, Boucher Y. Emergence, ecology and dispersal of the pandemic generating Vibrio cholerae lineage. International Microbiology Official journal of the Spanish Society for Microbiology. 2017;106-15. cholera pandemic. Proc Natl Acad Sci USA. 2016;113:E7730-9. Integrated view of Vibrio cholerae in the Americas. Science. 2017;358:789-93. history of the seventh pandemic of cholera in Africa. Science. 2017;358:785-9. Genomic insights into the 2016-2017 cholera epidemic in Yemen. Nature.

774 2019;565:230-3.

46. Unterweger D, Kitaoka M, Miyata ST, Bachmann V, Brooks TM, Moloney J, et al. Constitutive Type VI Secretion System Expression Gives Vibrio cholerae Intra- and Interspecific Competitive Advantages. Soldati T, editor. PLoS ONE. 2012;7:e48320. and Environmental Isolates of Vibrio cholerae in Natural Transformation and ContactDependent Bacterial Killing Indicative of Type VI Secretion System Activity. Liu S-J, editor. Appl Environ Microbiol. 2016;82:2833-42.

782 48. Crisan CV, Hammer BK. The VIBRIO CHOLERAE type VI secretion system: toxins, 783 regulators and consequences. Environ Microbiol. 2020;1462-2920.14976.

784 49. Miyata ST, Kitaoka M, Wieteska L, Frech C, Chen N, Pukatzki S. The Vibrio 785 Cholerae Type VI Secretion System: Evaluating its Role in the Human Disease Cholera. 
786 Front Microbio [Internet]. 2010 [cited 2020 Jul 26];1. Available from:

787 http://journal.frontiersin.org/article/10.3389/fmicb.2010.00117/abstract

788 50. Thomas J, Watve SS, Ratcliff WC, Hammer BK. Horizontal Gene Transfer of

789 Functional Type VI Killing Genes by Natural Transformation. Bassler B, editor. mBio. 2017;8:mBio.00654-17, e00654-17.

791 51. López-Pérez M, Jayakumar JM, Haro-Moreno JM, Zaragoza-Solas A, Reddi G, 792 Rodriguez-Valera F, et al. Evolutionary Model of Cluster Divergence of the Emergent 793 Marine Pathogen Vibrio vulnificus : From Genotype to Ecotype. Laub MT, editor. mBio. 794 2019;10:e02852-18, /mbio/10/1/mBio.02852-18.atom.

795

796

797

798

799

800

801

802

803

804

805

806

807

808

809

810

811

812

813

814

815

816

817

818

819

820

821

822

52. Haley BJ, Choi SY, Grim CJ, Onifade TJ, Cinar HN, Tall BD, et al. Genomic and Phenotypic Characterization of Vibrio cholerae Non-O1 Isolates from a US Gulf Coast Cholera Outbreak. Chang Y-F, editor. PLoS ONE. 2014;9:e86264.

53. Prudhomme M, Libante V, Claverys J-P. Homologous recombination at the border: insertion-deletions and the trapping of foreign DNA in Streptococcus pneumoniae. Proc Natl Acad Sci U S A. 2002;99:2100-5.

54. de Vries J, Wackernagel W. Integration of foreign DNA during natural transformation of Acinetobacter sp. by homology-facilitated illegitimate recombination. Proceedings of the National Academy of Sciences. 2002;99:2094-9.

55. Meier $P$, Wackernagel $W$. Mechanisms of homology-facilitated illegitimate recombination for foreign DNA acquisition in transformable Pseudomonas stutzeri. Mol Microbiol. 2003;48:1107-18.

56. Amarir-Bouhram J, Goin M, Petit M-A. Low Efficiency of Homology-Facilitated Illegitimate Recombination during Conjugation in Escherichia coli. Kirkpatrick DT, editor. PLoS ONE. 2011;6:e28876.

57. Nakajima T, Suzuki M, Harada K, Inoue M, Mitsuhashi S. Transmission of R Plasmids in Vibrio anguillarum to Vibrio cholerae. Microbiology and Immunology. 1983;27:195-8.

58. Morita D, Morita M, Alam M, Mukhopadhyay AK, Johura F-T, Sultana M, et al. Whole-Genome Analysis of Clinical Vibrio cholerae O1 in Kolkata, India, and Dhaka, Bangladesh, Reveals Two Lineages of Circulating Strains, Indicating Variation in Genomic Attributes. mBio. 2020;11.

59. Nguyen TH, Pham TD, Higa N, Iwashita H, Takemura T, Ohnishi M, et al. Analysis of Vibrio seventh pandemic island II and novel genomic islands in relation to attachment sequences among a wide variety of Vibrio cholerae strains. Microbiol Immunol. 2018;62:150-7.

60. Borgeaud S, Metzger L, Scrignari T, Blokesch M. The type VI secretion system of Vibrio cholerae fosters horizontal gene transfer. 2015;347:63-7. 
823 61. Lages MA, Balado M, Lemos ML. The Expression of Virulence Factors in Vibrio 824 anguillarum Is Dually Regulated by Iron Levels and Temperature. Front Microbiol.

$825 \quad$ 2019;10:2335.

826 62. O'Toole R, Lundberg S, Fredriksson S-A, Jansson A, Nilsson B, Wolf-Watz H. The Chemotactic Response of Vibrio anguillarum to Fish Intestinal Mucus Is Mediated by a 828 Combination of Multiple Mucus Components. J Bacteriol. 1999;181:4308-17.

829 63. Pedersen K, Grisez L, Houdt R van, Tiainen T, Ollevier F, Larsen JL. Extended 830 Serotyping Scheme for Vibrio anguillarum with the Definition and Characterization of 831 Seven Provisional O-Serogroups. Curr Microbiol. 1999;38:183-9.

832 64. Meibom KL. Chitin Induces Natural Competence in Vibrio cholerae. Science. 833 2005;310:1824-7.

834 65. Hunt DE, Gevers D, Vahora NM, Polz MF. Conservation of the Chitin Utilization 835 Pathway in the Vibrionaceae. AEM. 2008;74:44-51.

836 66. Halpern M, Izhaki I. Fish as Hosts of Vibrio cholerae. Front Microbiol [Internet]. 2017 [cited $2020 \mathrm{Jul} 26$ ];8. Available from:

838 http://journal.frontiersin.org/article/10.3389/fmicb.2017.00282/full

839 67. Halpern M, Senderovich Y, Izhaki I. Waterfowl: the missing link in epidemic and pandemic cholera dissemination? PLoS Pathog. 2008;4:e1000173.

841 68. Laviad-Shitrit S, Izhaki I, Halpern M. Accumulating evidence suggests that some 842 waterbird species are potential vectors of Vibrio cholerae. Bliska JB, editor. PLoS 843 Pathog. 2019;15:e1007814.

844 69. Runft DL, Mitchell KC, Abuaita BH, Allen JP, Bajer S, Ginsburg K, et al. Zebrafish as a natural host model for Vibrio cholerae colonization and transmission. Appl Environ 846 Microbiol. 2014;80:1710-7.

847 70. Mitchell KC, Breen P, Britton S, Neely MN, Withey JH. Quantifying Vibrio cholerae 848 Enterotoxicity in a Zebrafish Infection Model. Appl Environ Microbiol. 2017;83.

849 71. Logan SL, Thomas J, Yan J, Baker RP, Shields DS, Xavier JB, et al. The Vibrio cholerae type $\mathrm{VI}$ secretion system can modulate host intestinal mechanics to displace gut bacterial symbionts. Proc Natl Acad Sci USA. 2018;115:E3779-87.

852 72. Vezzulli L, Guzmán CA, Colwell RR, Pruzzo C. Dual role colonization factors connecting Vibrio cholerae's lifestyles in human and aquatic environments open new perspectives for combating infectious diseases. Current Opinion in Biotechnology. 855 2008;19:254-9.

856 73. Ma AT, Mekalanos JJ. In vivo actin cross-linking induced by Vibrio cholerae type VI 857 secretion system is associated with intestinal inflammation. Proceedings of the National 858 Academy of Sciences. 2010;107:4365-70. 
859 74. Seemann T. Prokka: rapid prokaryotic genome annotation. Bioinformatics.

860 2014;30:2068-9.

861 75. Edgar RC. MUSCLE: multiple sequence alignment with high accuracy and high

862 throughput. Nucleic Acids Research. 2004;32:1792-7.

863 76. Edgar RC. Search and clustering orders of magnitude faster than BLAST.

864 Bioinformatics. 2010;26:2460-1.

865 77. Chaudhari NM, Gupta VK, Dutta C. BPGA- an ultra-fast pan-genome analysis

866 pipeline. Sci Rep. 2016;6:24373.

867 78. Stamatakis A. RAxML version 8: a tool for phylogenetic analysis and post-analysis

868 of large phylogenies. Bioinformatics. 2014;30:1312-3.

869 79. Stöver BC, Müller KF. TreeGraph 2: Combining and visualizing evidence from

870 different phylogenetic analyses. BMC Bioinformatics. 2010;11:7.

871 80. Page AJ, Cummins CA, Hunt M, Wong VK, Reuter S, Holden MTG, et al. Roary:

872 rapid large-scale prokaryote pan genome analysis. Bioinformatics. 2015;31:3691-3.

873 81. Page AJ, Taylor B, Delaney AJ, Soares J, Seemann T, Keane JA, et al. SNP-sites:

874 rapid efficient extraction of SNPs from multi-FASTA alignments. Microbial Genomics

875 [Internet]. 2016 [cited 2021 May 14];2. Available from:

876 https://www.microbiologyresearch.org/content/journal/mgen/10.1099/mgen.0.000056

877 82. Li W, Godzik A. Cd-hit: a fast program for clustering and comparing large sets of 878 protein or nucleotide sequences. Bioinformatics. 2006;22:1658-9.

879 83. Fu L, Niu B, Zhu Z, Wu S, Li W. CD-HIT: accelerated for clustering the next-

880 generation sequencing data. Bioinformatics. 2012;28:3150-2.

881 84. Carver TJ, Rutherford KM, Berriman M, Rajandream M-A, Barrell BG, Parkhill J.

882 ACT: the Artemis Comparison Tool. Bioinformatics. 2005;21:3422-3.

883

884

885

886

887

888

889 
$891 \quad$ Figure 1

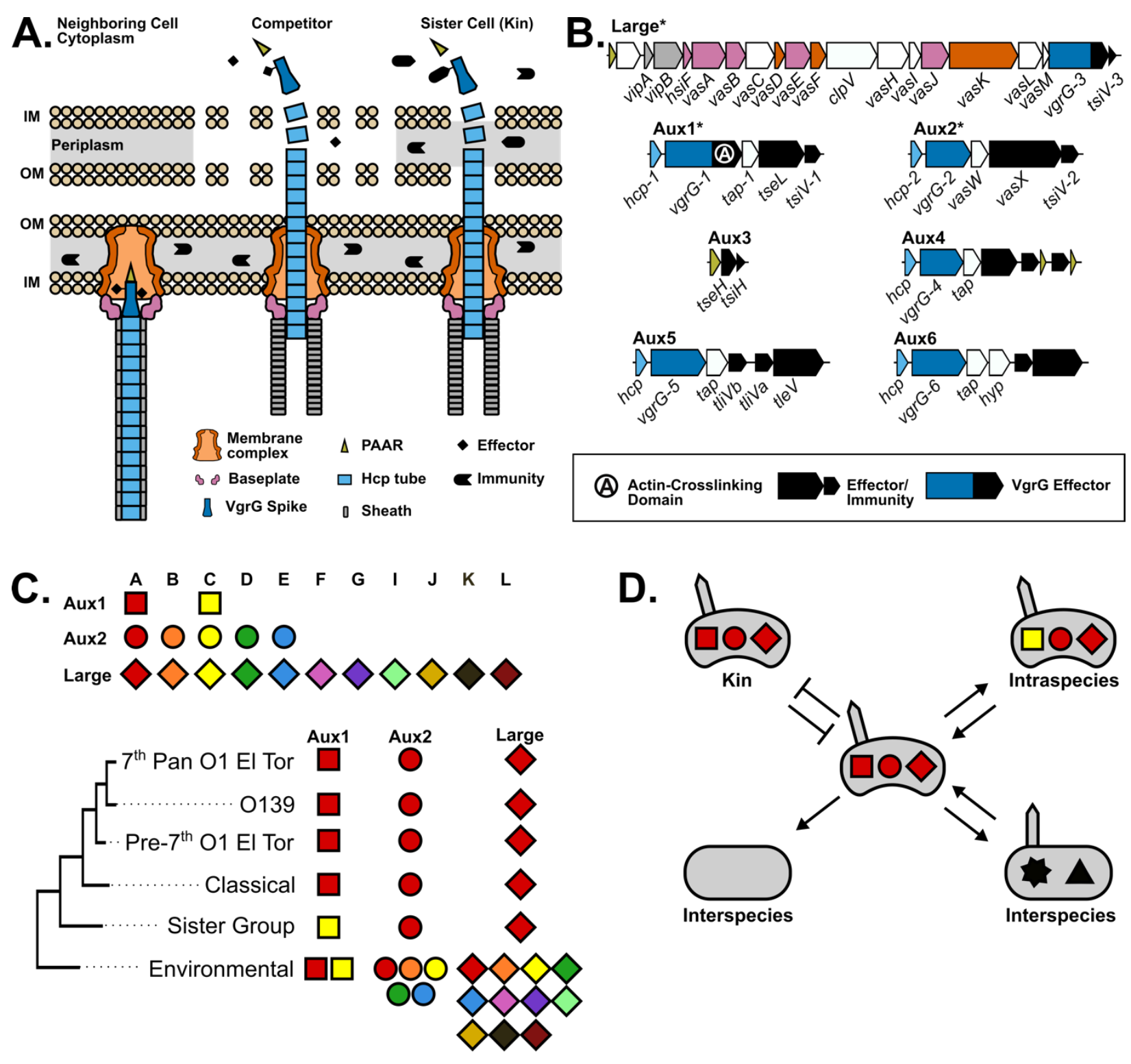
competition. with a neighboring non-kin (middle) or kin (right) cell. b Genetic diagrams of all known Vch T6SS

897 loci. Coding regions are colored according to their corresponding T6SS component in (a). White 898 coding regions are not represented in (a). Core T6SS loci are marked with an *. c Known V. 
bioRxiv preprint doi: https://doi.org/10.1101/2021.05.28.446156; this version posted May 28, 2021. The copyright holder for this preprint (which

was not certified by peer review) is the author/funder, who has granted bioRxiv a license to display the preprint in perpetuity. It is made available under aCC-BY-NC-ND 4.0 International license.

899 cholerae effector types and their distribution across different $V$. cholerae clades. d Theoretical

900 schematic of T6SS-based competition for cells with identical and differential T6SS effector sets.

901

902

903

904

905

906

907

908

909

910

911

912

913

914

915

916

917

918

919

920

921

922

923

924 
bioRxiv preprint doi: https://doi.org/10.1101/2021.05.28.446156; this version posted May 28, 2021. The copyright holder for this preprint (which was not certified by peer review) is the author/funder, who has granted bioRxiv a license to display the preprint in perpetuity. It is made available under aCC-BY-NC-ND 4.0 International license.

Figure 2

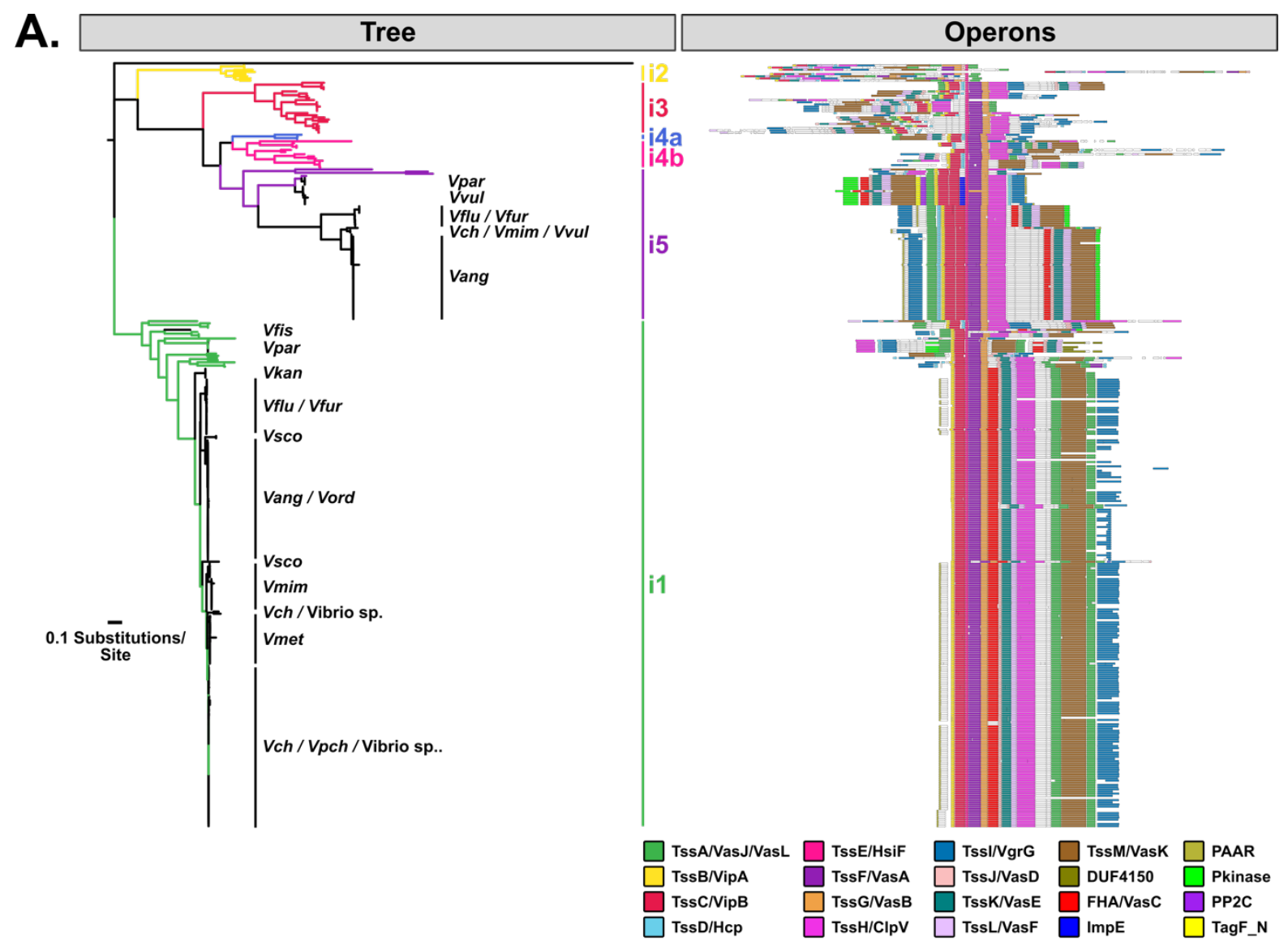

B.

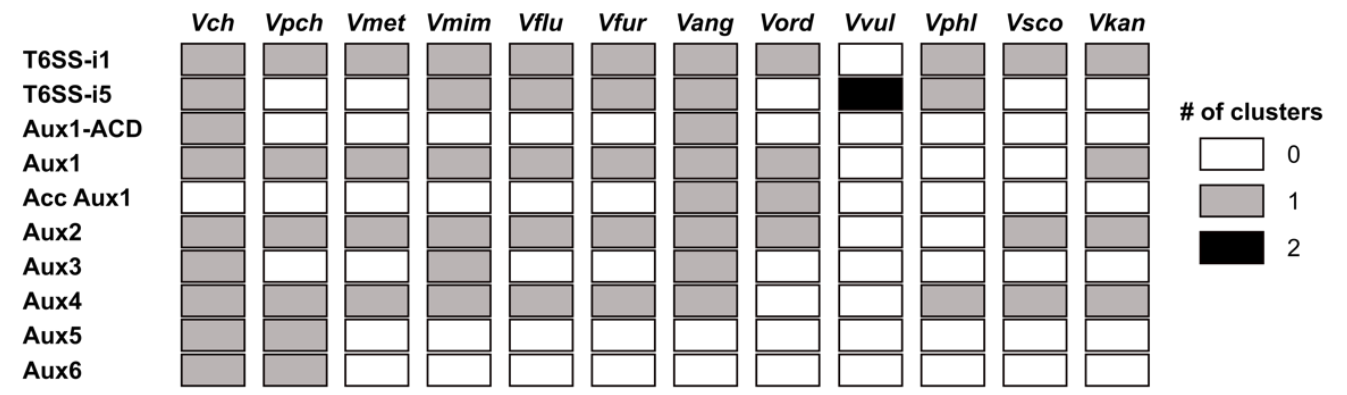

C. Actin-Crosslinking Aux1

Aux1/Large Hybrid

Accessory Aux1

- $v$ vgrG-1 \&

Aux3 Aux4

Aux2

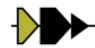

Aux4

Large (T6SS-i1) and Large/Aux1 Hybrids

$-\rightarrow>>>>>>>(7)$ 
928 V. anguillarum encodes most known V. cholerae T6SS loci and a single unique T6SS

929 cluster.

930 a Hamburger ((https://github.com/djw533/hamburger) analysis of all analyzed Vibrio genomes

931 showing all identified complete T6SS clusters. Grouping of the identified T6SS clusters with

932 known T6SS types is shown by phylogenetic tree (left) and operon structure is indicated (right).

933 Colored branches of the tree indicate Hamburger-internal representative T6SSs corresponding

934 to the indicated type, and black branches indicate input genomes. Operon diagrams are

935 centered around vipA/vipB. b Presence-absence heatmap of all T6SS clusters identified in $V$.

936 cholerae and V. anguillarum and their distribution across all analyzed Vibrio species. c

937 Schematic diagrams of T6SS clusters identified in V. anguillarum showing known V. cholerae

938 clusters, V. anguillarum-specific hybrid clusters, and a unique V. anguillarum locus (Accessory

939 Aux1).

940

941

942

943

944

945

946

947

948

949

950

951

952

953 


\section{Figure 3}

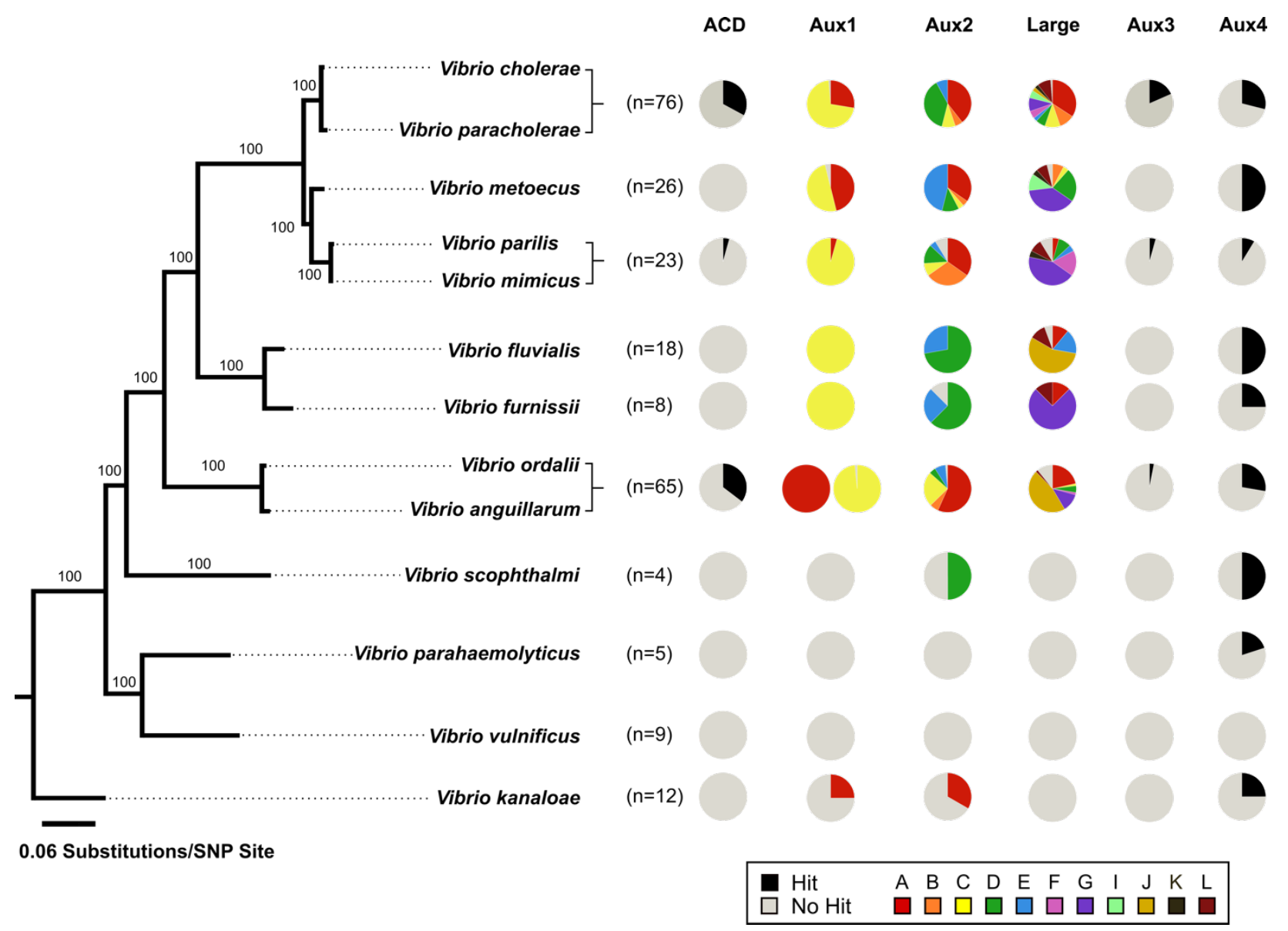

Known V. cholerae T6SS effector types, including pandemic-associated effectors, are

958 (Left) Maximum-likelihood tree built on 1,889 core proteins from 30 Vibrio genomes collapsed by

959 species. Branch support was calculated using 100 bootstrap replicates. Support values are

960 indicated. (Right) Pie charts indicating the presence of each known V. cholerae Aux1, Aux2, and

961 Large effector type (Fig. 1c) as well as the actin-crosslinking domain of vgrG-1, the Aux3

962 effector $t s e H$ and the Aux4 effector tpeV. Number of genomes included for each species $(n)$ is

963 indicated. 
bioRxiv preprint doi: https://doi.org/10.1101/2021.05.28.446156; this version posted May 28, 2021. The copyright holder for this preprint (which

was not certified by peer review) is the author/funder, who has granted bioRxiv a license to display the preprint in perpetuity. It is made available under aCC-BY-NC-ND 4.0 International license.

\section{$967 \quad$ Figure 4}

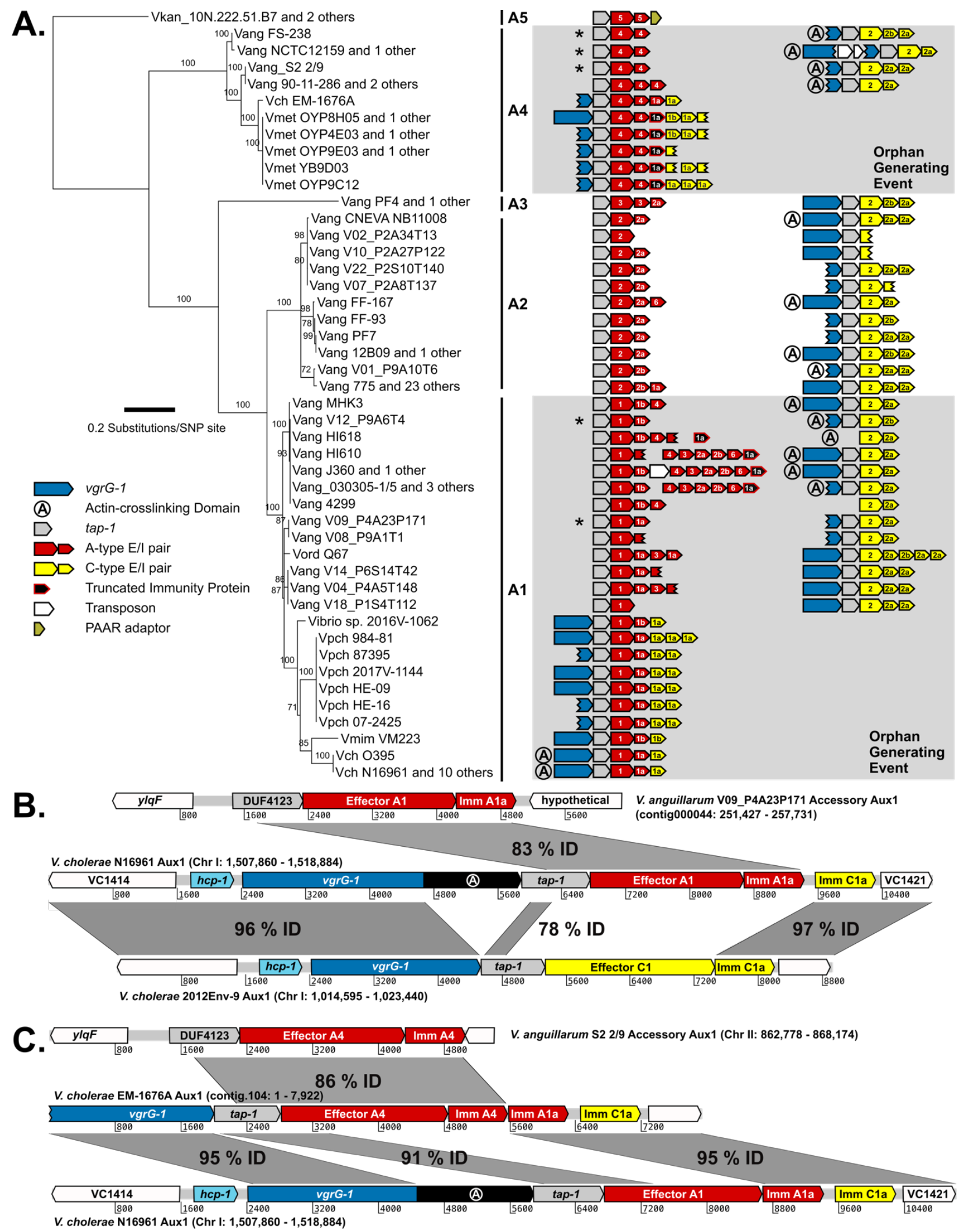


970 strains.

971 a Maximum-likelihood tree of identified Aux1 A-type effectors (TseL). Bootstrapping support

972 values for each node are shown. Accessory Aux1 and/or Aux1 schematic for each strain is

973 shown on the right. Effector/immunity type is indicated by color, and each effector/immunity

974 cassette is labeled with its associated subtype. Grey boxes indicate groups of strains potentially

975 involved in transfer of the A-type effector from V. anguillarum and the generation of an orphan

976 immunity gene in $V$. cholerae. Asterisks mark the V. anguillarum Accessory Aux1 clusters most

977 likely to have been transferred to V. cholerae. b Artemis alignment of the T6SS Aux1 cluster

978 from pandemic V. cholerae strain N16961 with its potential precursor loci - Accessory Aux1

979 from V. anguillarum V09_P4A23P171 and Aux1 from V. cholerae 2012Env-9. This alignment

980 represents the potential orphan generating transfer event indicated in (a, bottom grey box). c

981 Artemis alignment of the T6SS Aux1 cluster from V. cholerae strain EM-1676A with its potential

982 precursor loci - Accessory Aux1 from V. anguillarum S2 2/9 and Aux1 from V. cholerae

983 N16961. This alignment represents the potential orphan generating transfer event indicated in

984 (a, top grey box). For Artemis alignments, regions of homology are indicated by grey boxes,

985 which are labeled with the nucleotide identity between the covered regions. 

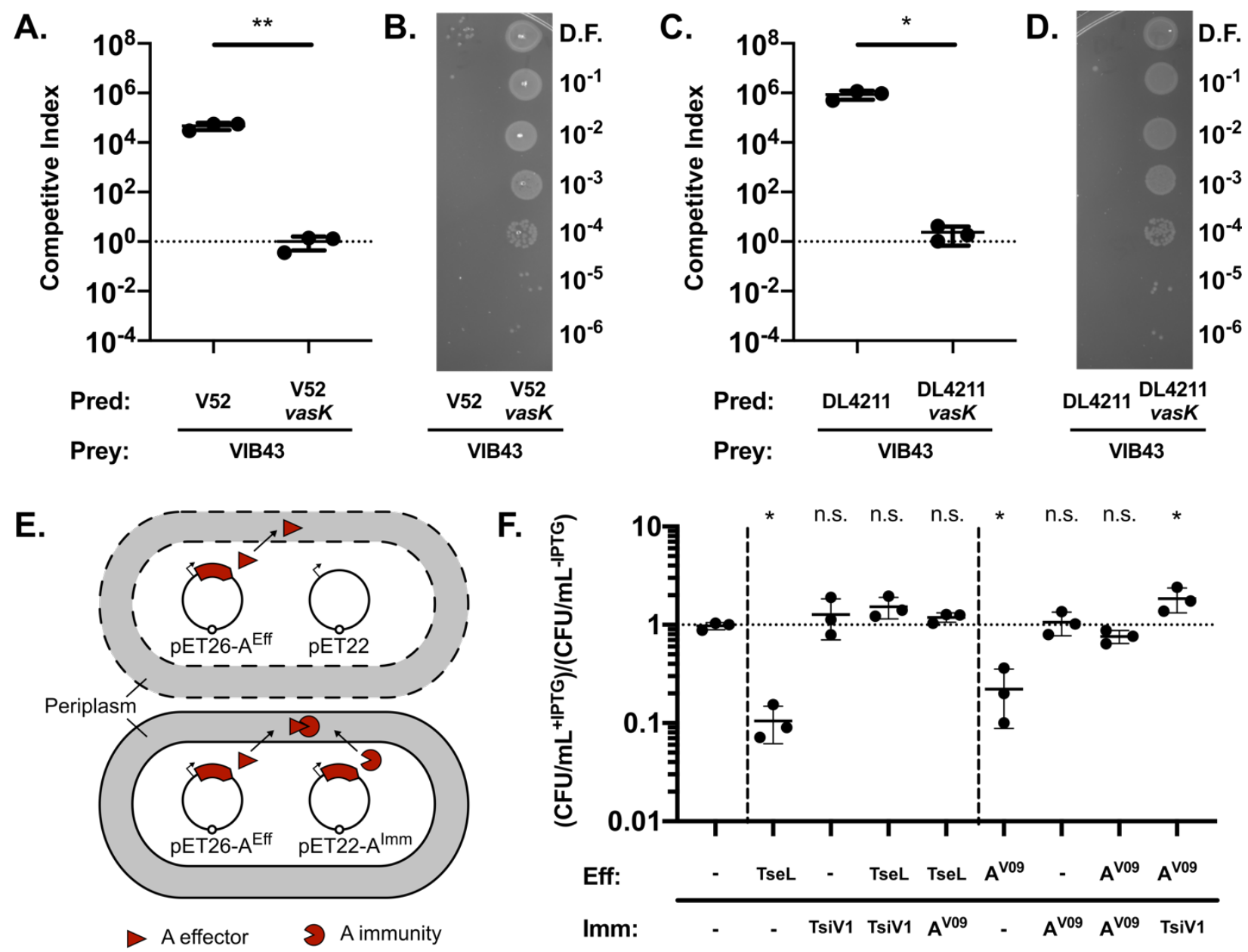

998 A-type effector/immunity pairs from V. cholerae Aux1 and V. anguillarum Accessory

999 Aux1 are cross-neutralizing.

1000 a Competitive killing assay results for V. cholerae strain V52 (T6SS on) or V52 $\Delta$ vasK (T6SS

1001 off) vs V. anguillarum strain VIB43. b Representative image of dilution spots on agar plates

1002 selecting for VIB43 prey cells from competition assays against V52 or V52 $\Delta$ vasK. c Competitive

1003 killing assay results for $V$. cholerae strain DL4211 (T6SS on) or DL4211 $\Delta$ vasK (T6SS off) vs V.

1004 anguillarum strain VIB43. d Representative image of dilution spots on agar plates selecting for

1005 VIB43 prey cells from competition assays against DL4211 or DL4211 $\Delta$ vasK. a,c Significance

1006 was determined by unpaired t test $\left({ }^{*} p=0.0115,{ }^{* *} p=0.0056\right)$. e Diagram of E. coli BL21(DE3) 
1007 dual-expression viability assay. Dashed cell membranes represent lysis. $\mathbf{f}$ Dual-expression

1008 viability assay with results plotted as the ratio of CFU/mL recovered with induction (+IPTG) to

$1009 \mathrm{CFU} / \mathrm{mL}$ recovered without induction (-IPTG). TseL $=$ V. cholerae A1 effector. TsiV1 $=$ V.

1010 cholerae $\mathrm{A} 1 \mathrm{a}$ immunity protein. $\mathrm{A}^{\mathrm{V} 09}=\mathrm{V}$. anguillarum $\mathrm{A} 1$ effector or $\mathrm{A} 1 \mathrm{a}$ immunity protein.

1011 Significance was determined by one-way ANOVA with Dunnet's multiple comparisons test $\left({ }^{*} p=\right.$

$1012(0.0190,0.0486,0.0182)$, n.s. $=$ non-significant $)$. All comparisons were made to strain carrying

1013 two empty vectors. a,c,f Quantitative results are from three independent experiments $(n=3)$.

1014 Individual replicates are shown. Horizontal bars represent the mean, and error bars represent

1015 SD.

1016

1017

1018

1019

1020

1021

1022

1023

1024

1025

1026

1027

1028

1029

1030

1031

1032 
A.
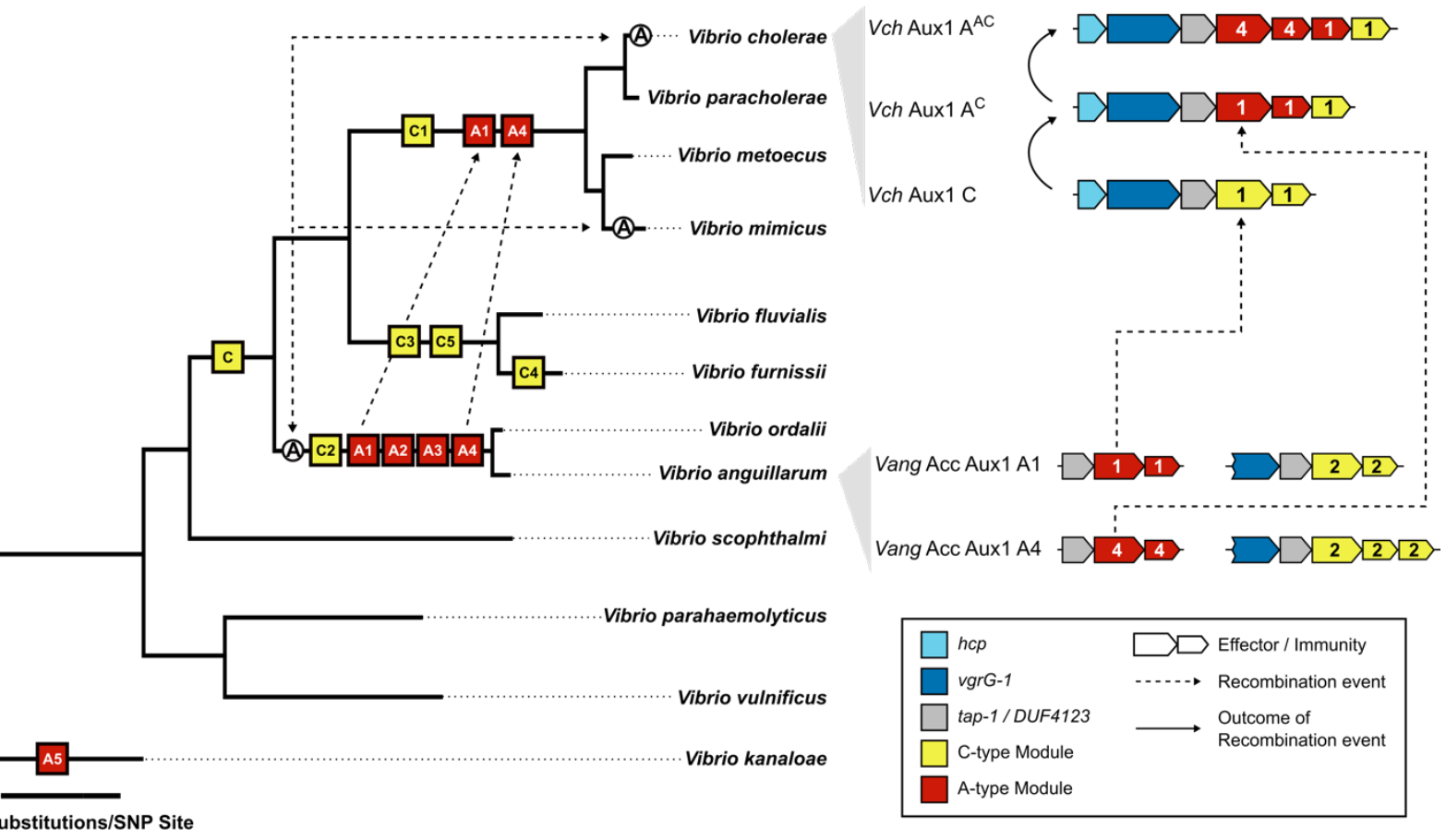

0.06 Substitutions/SNP Site

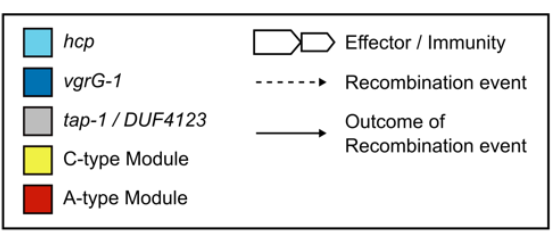

\section{B. Minimal Scenario}

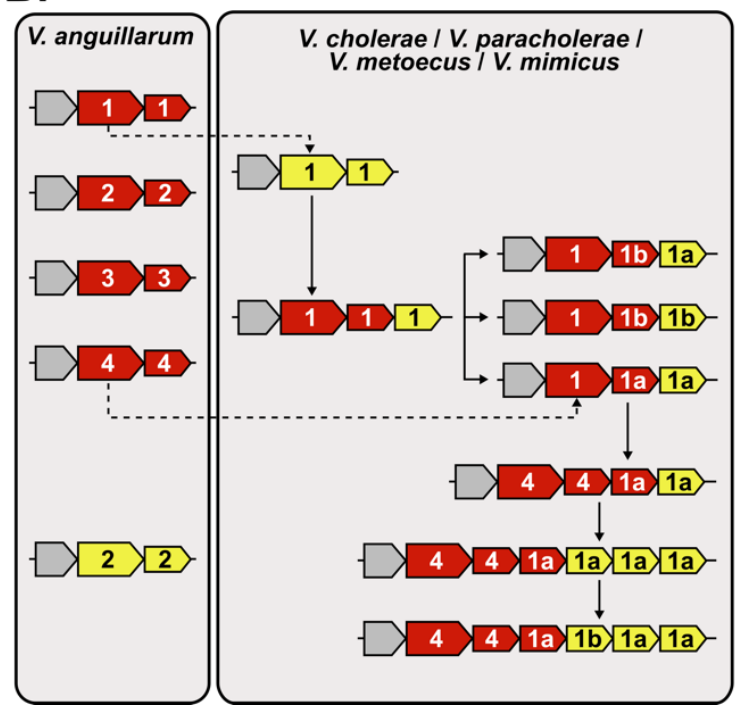

C. Maximal Scenario

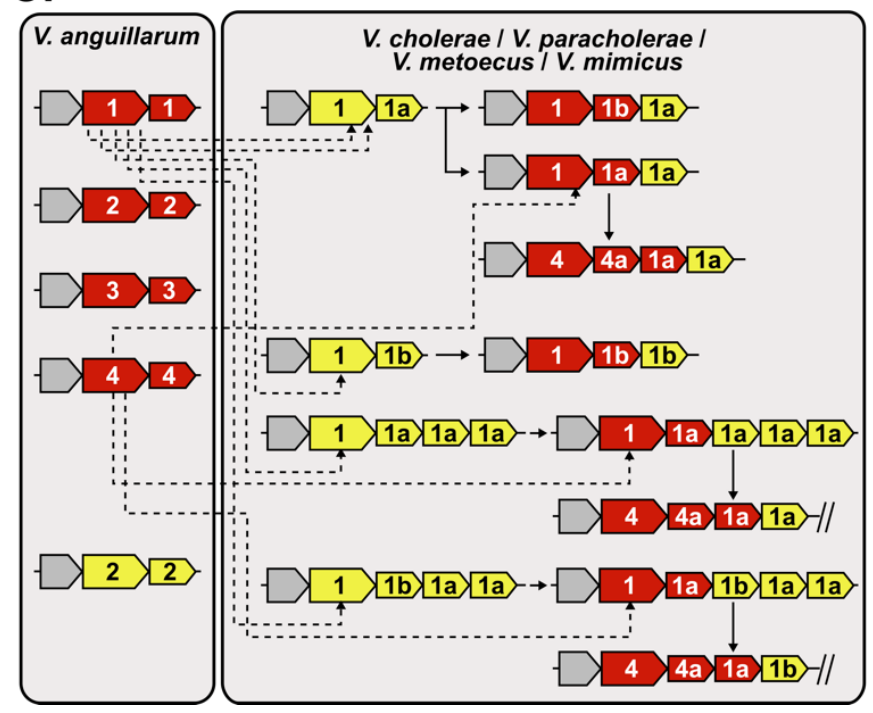


1040 Representative genetic diagrams of Aux1 clusters in V. cholerae as well as Accessory Aux1 and

1041 Aux1 clusters in V. anguillarum. Effector/immunity types are indicated by color and

1042 effector/immunity subtypes are indicated. b,c Schematic diagrams representing the potential

1043 transfer events leading to the array of Aux1 clusters observed in V. cholerae, V. paracholerae,

1044 V. metoecus, and V. mimicus. b Minimal gene transfer scenario consisting of Aux1 A1 transfer,

1045 divergence of the recipient cluster, subsequent transfer of Aux1 A4, and further divergence of

1046 that cluster. c Maximal gene transfer scenario consisting of multiple individual transfer events

1047 for both Aux1 A1 and Aux1 A4. a-c Effector/immunity types are indicated by color and

1048 effector/immunity subtypes are indicated. Dashed arrows indicate recombination events, and

1049 solid arrows indicate the resulting cluster from said recombination events. 